\title{
Deficient Values of Solutions of Linear Differential Equations
}

\author{
Gary G. Gundersen ${ }^{1}$ • Janne Heittokangas ${ }^{2,4}$ - Zhi-Tao Wen ${ }^{3,4}$
}

Received: 21 June 2019 / Revised: 10 March 2020 / Accepted: 20 March 2020 /

Published online: 12 May 2020

(c) The Author(s) 2020

\begin{abstract}
Differential equations of the form $f^{\prime \prime}+A(z) f^{\prime}+B(z) f=0(*)$ are considered, where $A(z)$ and $B(z) \not \equiv 0$ are entire functions. The Lindelöf function is used to show that for any $\rho \in(1 / 2, \infty)$, there exists an equation of the form $\left(^{*}\right)$ which possesses a solution $f$ with a Nevanlinna deficient value at 0 satisfying $\rho=\rho(f) \geq \rho(A) \geq \rho(B)$, where $\rho(h)$ denotes the order of an entire function $h$. It is known that such an example cannot exist when $\rho \leq 1 / 2$. For smaller growth functions, a geometrical modification of an example of Anderson and Clunie is used to show that for any $\rho \in(2, \infty)$, there exists an equation of the form $(*)$ which possesses a solution $f$ with a Valiron deficient value at 0 satisfying $\rho=\rho_{\log }(f) \geq \rho_{\log }(A) \geq \rho_{\log }(B)$, where $\rho_{\log }(h)$ denotes the logarithmic order of an entire function $h$. This result is essentially sharp. In both proofs, the separation of the zeros of the indicated solution plays a key role. Observations on the deficient values of solutions of linear differential equations are also given, which include a discussion of Wittich's theorem on Nevanlinna deficient values, a modified Wittich theorem for Valiron deficient values, consequences of Gol'dberg's theorem, and examples to illustrate possibilities that can occur.
\end{abstract}

Keywords Finite order · Logarithmic order - Linear differential equation ·

Nevanlinna deficient value $\cdot$ Valiron deficient value

Mathematics Subject Classification Primary 34M05 - Secondary 34M10 · 30D35

\section{Introduction}

The solutions of the linear differential equation

$$
f^{(n)}+A_{n-1}(z) f^{(n-1)}+\cdots+A_{1}(z) f^{\prime}+A_{0}(z) f=0
$$

Communicated by James K. Langley.

Extended author information available on the last page of the article 
with entire coefficients $A_{0}(z), \ldots, A_{n-1}(z), A_{0}(z) \not \equiv 0$, are entire, and it is well known that the zeros of any solution $f \not \equiv 0$ of (1.1) are of multiplicity $\leq n-1$. The main focus of this paper is on solutions of (1.1) which have less than the usual frequency of zeros. A standard measurement of the frequency of a $c$-point $(c \in \mathbb{C})$ of an entire function $f$ is the Nevanlinna deficiency $\delta_{N}(c, f)$ defined by

$$
\delta_{N}(c, f)=\liminf _{r \rightarrow \infty} \frac{m(r, f, c)}{T(r, f)}=1-\limsup _{r \rightarrow \infty} \frac{N(r, f, c)}{T(r, f)} .
$$

If $\delta_{N}(c, f)>0$, then $c$ is said to be a Nevanlinna deficient value of $f$.

Let $\rho(f)$ denote the order of an entire function $f$. It is known [6, p. 207] that an entire function $f$ cannot possess a finite Nevanlinna deficient value when $\rho(f) \leq 1 / 2$. Thus it can be asked:

For any given $\rho \in(1 / 2, \infty)$, does there exist an equation of the form (1.1) that possesses a solution $f$ satisfying $\rho(f)=\rho$ and $\delta_{N}(0, f)>0$ ?

If there are no restrictions on the orders of the coefficients $A_{j}(z)$ in (1.1), then Gol'dberg's theorem (stated in Sect. 3) could be used to easily answer this question affirmatively, see the sentence following (1.3). By putting a common growth restriction on the coefficients, the following result answers this question for second order equations

$$
f^{\prime \prime}+A(z) f^{\prime}+B(z) f=0,
$$

where $A(z)$ and $B(z) \not \equiv 0$ are entire functions.

Theorem 1 For any given $\rho \in(1 / 2, \infty)$, there exists an equation of the form (1.2) which possesses a solution $f$ satisfying $\delta_{N}(0, f)>0$ and $\rho=\rho(f) \geq \rho(A) \geq \rho(B)$.

Theorem 1 is sharp with respect to $\rho \in(1 / 2, \infty)$ because, as noted above, the result does not hold for $\rho \leq 1 / 2$. The solution $f$ in the proof of Theorem 1 is the classical Lindelöf function $L_{\rho}$ in (6.1). Thus the crux of the proof is to find entire coefficients $A(z)$ and $B(z)$ satisfying (1.2) and

$$
\rho(f) \geq \rho(A) \geq \rho(B) .
$$

If the inequalities (1.3) were removed from the conclusion of Theorem 1, then Theorem 1 would easily follow by combining Gol'dberg's theorem with the known properties that $L_{\rho}$ has all simple zeros and $\delta_{N}\left(0, L_{\rho}\right)>0$, see Sects. 3 and 6 . The proof of Theorem 1 involves proving a separation of zeros property of $L_{\rho}$, namely, that the zeros of $L_{\rho}$ are uniformly $q$-separated for any $q>\rho$.

Regarding (1.3), recall that (1.2) cannot possess a non-trivial solution of finite order in the case when $\rho(A)<\rho(B)$, see [8, Thm. 2]. Many examples in the literature of solutions of (1.2) involve elementary functions, where $A(z), B(z), f$ are of integer order and satisfy the double inequalities (1.3). However, there are examples of $A(z), B(z), f$ that are of integer order and still do not satisfy (1.3), as Examples 2 and 8 
below show. In addition, Theorem 1 addresses all the orders in the infinite interval $(1 / 2, \infty)$, not just the integer orders.

Example 2 If $w(z)$ is any entire function, then $f(z)=e^{z}$ satisfies

$$
f^{\prime \prime}+w(z) f^{\prime}-(1+w(z)) f=0 .
$$

For smaller growth functions $f$, we obtain an analogous result to Theorem 1 by considering Valiron deficient values and logarithmic order. The Valiron deficiency $\delta_{V}(c, f)$ of a $c$-point of an entire function $f$ is defined by

$$
\delta_{V}(c, f)=\limsup _{r \rightarrow \infty} \frac{m(r, f, c)}{T(r, f)}=1-\liminf _{r \rightarrow \infty} \frac{N(r, f, c)}{T(r, f)} .
$$

Clearly $0 \leq \delta_{N}(c, f) \leq \delta_{V}(c, f) \leq 1$. If $\delta_{V}(c, f)>0$, then $c$ is said to be a Valiron deficient value of $f$.

For slowly growing entire functions, finite Nevanlinna deficient values are not possible but Valiron deficient values are possible. The growth of such functions $f$ can be measured in terms of the logarithmic order $\rho_{\log }(f)$ defined by

$$
\rho_{\log }(f)=\limsup _{r \rightarrow \infty} \frac{\log T(r, f)}{\log \log r} .
$$

Observe that finite logarithmic order implies zero order, non-constant polynomials have logarithmic order one, and that there do not exist any non-constant entire functions of logarithmic order $<1$, see $[3,4]$.

A classical result of Valiron [1,21] says that any entire function $f$ satisfying $T(r, f)=O\left(\log ^{2} r\right)$ has no finite Valiron deficient values. Entire functions $f$ satisfying $\rho_{\log }(f)<2$ have this growth rate. Hence we state our main result regarding Valiron deficient values as follows.

Theorem 3 For any given $\rho \in(2, \infty)$, there exists an equation of the form (1.2) which possesses a solution $f$ satisfying $\delta_{V}(0, f)=1$ and $\rho=\rho_{\log }(f) \geq \rho_{\log }(A) \geq$ $\rho_{\log }(B)$, where $A(z)$ is transcendental.

Theorem 3 is essentially sharp with respect to $\rho \in(2, \infty)$ because the result does not hold when $\rho<2$, and the only unsettled case is when $\rho_{\log }(f)=2$ and $f$ has infinite logarithmic type.

The solution $f$ in Theorem 3 is a laborious modification of a function due to Anderson and Clunie [1], and its symmetric geometric construction takes a substantial portion of this paper. More precisely, the proof of [1, Thm. 2] involves a canonical product with negative real zeros of unbounded multiplicity. Such a function is a real entire function (real on reals), but it cannot be a solution of (1.2) because it has zeros with unbounded multiplicities. Thus we modify the reasoning in [1] in such a way that the revised canonical product $f$ has only simple zeros lying symmetrically in the left half-plane, where $f$ has the pre-determined logarithmic order. The zeros of $f$ will be pairs of complex conjugates so that $f$ becomes real entire, and, in addition, 
$f(r)=M(r, f)$ holds. These properties are crucial for proving that $\delta_{V}(0, f)=1$. A consequence of the proof of Theorem 3 is that the zeros of the modified AndersonClunie function $f$ are uniformly logarithmically $q$-separated for any $q>\rho_{\log }(f)-1$.

After preparations in Sect. 5, we prove Theorem 1 in Sect. 6, while after preparations in Sect. 7, we prove Theorem 3 in Sect. 8. For convenience, Sect. 4 contains information about the separation of zeros of entire functions.

In Sect. 3, we discuss the possibilities for the sets $E_{N}(f)$ and $E_{V}(g)$ of the Nevanlinna and Valiron deficiencies of solutions $f, g$ of equations of the general form (1.1). It is shown that it follows from Gol'dberg's theorem that $E_{N}(f)$ can be countably infinite and $E_{V}(g)$ can be uncountable. The next section contains examples that illustrate a classical theorem of Wittich on Nevanlinna deficient values and a particular modified Wittich theorem for Valiron deficient values.

\section{Wittich's Theorem}

On the topic of possible deficiencies of solutions of equations of the form (1.1), we recall the following well-known result of Wittich on Nevanlinna deficient values.

Wittich's theorem ([15, Thm. 4.3], [22]) Suppose that a solution f of (1.1) is admissible in the sense that

$$
T\left(r, A_{j}\right)=o(T(r, f)), \quad j=0, \ldots, n-1,
$$

as $r \rightarrow \infty$ outside a possible exceptional set of finite linear measure. Then 0 is the only possible finite Nevanlinna deficient value for $f$. In particular, this is true for transcendental solutions of (1.1) with polynomial coefficients.

The assumption on admissibility of $f$ cannot be removed in Wittich's theorem. The next example shows that if the growth of at least one of the coefficients in (1.1) is at least that of a solution $f$, then any $c \in \mathbb{C}$ can be a Picard value of $f$.

Example 4 For an arbitrary $c \in \mathbb{C}$ and an arbitrary entire function $w(z)$, the function $f(z)=e^{z}+c$ with $c$ as its Picard value solves the equation

$$
f^{(4)}+c e^{-z} f^{\prime \prime \prime}+w(z) f^{\prime \prime}-w(z) f^{\prime}-f=0 .
$$

The following example shows that 0 may or may not be a deficient value for an admissible solution.

Example 5 The function $f(z)=\exp \left(z^{2} / 2\right)$ is an admissible solution of

$$
f^{\prime \prime \prime}+e^{z} f^{\prime \prime}+\left(e^{z}-z e^{z}-1-z^{2}\right) f^{\prime}-\left(z e^{z}+e^{z}+2 z\right) f=0,
$$

which has 0 as a deficient value. On the other hand, from [9, Ex. 5], the function $g(z)=\exp \left(z^{2}\right)+e^{z}$ is an admissible solution of

$$
\begin{aligned}
f^{(4)} & +\left(8 z^{3}-13\right) f^{\prime \prime}-\left(16 z^{4}+16 z^{3}+12 z^{2}+4 z+2\right) f^{\prime} \\
& +\left(16 z^{4}+8 z^{3}+12 z^{2}+4 z+14\right) f=0 .
\end{aligned}
$$


We have $T(r, g) \sim N(r, 1 / g)$ as $r \rightarrow \infty$ by [19, Satz 1-2], so that $\delta_{N}(0, g)=0$. Observe that $f_{1}(z)=\exp \left(z^{2}\right)$ and $f_{2}(z)=e^{z}$ also satisfy (2.2), where $\delta_{N}\left(0, f_{1}\right)=$ $\delta_{N}\left(0, f_{2}\right)=1$.

The next example gives an equation of the form (1.2) whose non-trivial solutions are all admissible solutions with 0 as a deficient value.

Example 6 The functions $f(z)=\exp \left(z^{2} / 2\right) \sin z$ and $g(z)=\exp \left(z^{2} / 2\right) \cos z$ discussed in $[8$, p. 416] are linearly independent admissible solutions of

$$
f^{\prime \prime}-2 z f^{\prime}+z^{2} f=0
$$

It follows that all the non-trivial solutions of (2.3) are admissible and have 0 as a deficient value. Moreover, if $w(z)$ is any entire function, then $f$ and $g$ satisfy

$$
f^{\prime \prime \prime}+(w(z)-2 z) f^{\prime \prime}+\left(z^{2}-2-2 z w(z)\right) f^{\prime}+\left(z^{2} w(z)+2 z\right) f=0
$$

More examples of this kind can be generated by using [8, Ex. 4].

Next we note that the above examples for Nevanlinna deficient values are also examples for Valiron deficient values, since we always have $\delta_{N}(c, f) \leq \delta_{V}(c, f)$. Although the set $E_{N}(f)$ is at most countable from the second fundamental theorem, the set $E_{V}(f)$ can be uncountable. A classical result of Ahlfors-Frostman [18, p. 276] shows that $E_{V}(f)$ always has logarithmic capacity zero. Improvements of this result are due to Hyllengren [14] in the finite order case and to Hayman [12] in the infinite order case. See also [6, Ch. 4].

Wittich's theorem can easily be modified to deal with Valiron deficient values. To achieve this, we need to avoid all exceptional sets, and thus the reasoning works only for finite-order solutions. This modified result is particularly valuable when $\rho(f) \leq 1 / 2$, as we know that $f$ does not have finite Nevanlinna deficient values in this case. Solutions of zero-order are also possible, provided that at least one of the coefficients is transcendental [9]. For the convenience of the reader, a proof is given.

Modified Wittich's theorem Suppose that a finite-order solution $f$ of (1.1) satisfies (2.1) as $r \rightarrow \infty$ without an exceptional set. Then 0 is the only possible finite Valiron deficient value for $f$.

Proof Let $c \in \mathbb{C} \backslash\{0\}$. Write (1.1) in the form

$$
(f-c)^{(n)}+A_{n-1}(z)(f-c)^{(n-1)}+\cdots+A_{0}(z)(f-c)=-c A_{0}(z),
$$

which in turn can be written as

$$
\frac{1}{f-c}=-\frac{1}{c A_{0}(z)}\left(A_{0}(z)+A_{1}(z) \frac{(f-c)^{\prime}}{f-c}+\cdots+\frac{(f-c)^{(n)}}{f-c}\right) .
$$

Using a version of the lemma on the logarithmic derivative valid for functions of finite order (where no exceptional set appears) together with the first main theorem 
and the assumption (2.1) without an exceptional set, we obtain that $m(r, f, c)=$ $O(\log r)+o(T(r, f))$, as $r \rightarrow \infty$ without an exceptional set. The property (2.1) guarantees that $f$ is transcendental, even if the coefficients are polynomials. Thus, $m(r, f, c)=o(T(r, f))$ as $r \rightarrow \infty$ without an exceptional set. This proves that $c$ is not a Valiron deficient value of $f$.

Theorems 1 and 3 address a different question than that in Wittich's theorem and the modified Wittich's theorem. That said, we mention for independent interest that the proofs of Theorems 1 and 3 do not reveal whether the indicated solution $f$ is an admissible solution of (1.2) or not.

\section{Gol'dberg's Theorem and Sets of Deficiencies}

Any zero of a non-trivial solution of (1.1) must have multiplicity $\leq n-1$. Conversely, we have the following result.

Gol'dberg's theorem [10, p. 300] Let $f \not \equiv 0$ be an entire function whose zeros all have multiplicity at most $n-1, n \in \mathbb{N}$. Then $f$ is a solution of some differential equation of the form (1.1).

If $n=1$, then $f$ has no zeros, and the proof of Gol'dberg's theorem is trivial. For the convenience of the reader, we prove the case $n=2$.

Proof of Gol'dberg's theorem for $n=2$. Let $f \not \equiv 0$ be any entire function whose zeros are all simple. We assume that $f$ has at least one zero, since otherwise the proof is trivial.

We construct entire functions $A(z)$ and $B(z) \not \equiv 0$ such that $f$ solves (1.2). For $A(z)$ to be entire, at the zeros $z_{k}$ of $f, A(z)$ needs to solve the interpolation problem

$$
A\left(z_{k}\right)=-\frac{f^{\prime \prime}\left(z_{k}\right)}{f^{\prime}\left(z_{k}\right)}=\sigma_{k},
$$

where $\sigma_{k} \in \mathbb{C}$. Note that (3.1) can always be solved: If $\left\{z_{k}\right\}$ is a finite sequence, then $A(z)$ can be chosen to be the Lagrange interpolation polynomial, while if $\left\{z_{k}\right\}$ is an infinite sequence, then $A(z)$ can be constructed by means of Mittag-Leffler series. Let $\zeta \neq z_{k}$ be fixed. Along with (3.1), we may require that

$$
A(\zeta) \not \equiv-\frac{f^{\prime \prime}(\zeta)}{f^{\prime}(\zeta)}
$$

This guarantees that $f^{\prime \prime}(z)+A(z) f^{\prime}(z) \not \equiv 0$. After an entire $A(z)$ satisfying (3.1) and (3.2) has been found, we define $B(z)$ by

$$
B(z)=-\left(f^{\prime \prime}(z)+A(z) f^{\prime}(z)\right) / f(z),
$$

which is entire and $\not \equiv 0$. This completes the proof. 
Remark 1 If $f \not \equiv 0$ is any entire function, then for a suitable constant $c$, the function $g=f-c$ has all simple zeros. A consequence of this easy observation is that many properties of $f$, such as the number of deficient values, remain valid for $g$, and by Gol'dberg's theorem, $g$ solves some equation of the form (1.2).

Example 7 (1) Solutions of (1.2) may have any pre-given finite number $q \geq 2$ of Nevanlinna deficient values. Indeed, set

$$
f(z)=\int_{0}^{z} e^{-\zeta^{q}} d \zeta \text { and } a_{k}=e^{2 \pi k i / q} \int_{0}^{\infty} e^{-\zeta^{q}} d \zeta
$$

where $k=1, \ldots, q$. Then $f$ is entire, $\delta_{N}\left(a_{k}, f\right)=1 / q$, and $\delta_{N}(c, f)=0$ whenever $c \neq a_{k}, k=1, \ldots, q$, see [11, pp. 46-47]. Since $f^{\prime}(z)=e^{-z^{q}}$ has no zeros, we obtain that for any $c \neq a_{k},(k=1, \ldots, q)$, the function $g=f-c$ has all simple zeros and exactly $q$ Nevanlinna deficient values, and by Gol'dberg's theorem, $g$ is a solution of some equation of the form (1.2).

(2) Eremenko [6, p. 132] proved that for any countable set $E \subset \mathbb{C}$ and any $\rho>1 / 2$, there exists an entire function $f$ of order $\rho$ for which $E_{N}(f)=E$. If $f$ is any such function, then for a suitable $c \in \mathbb{C}$, the function $g=f-c$ has only simple zeros and countably many Nevanlinna deficient values, and $g$ solves an equation of the form (1.2).

(3) Let $f$ be an entire function with uncountably many Valiron deficient values [6, p. 118]. For a suitable $c \in \mathbb{C}, g=f-c$ has only simple zeros and uncountably many Valiron deficient values, and $g$ solves an equation of the form (1.2).

Gol'dberg's theorem does not give information about the orders of the coefficients in (1.1). Thus, although Gol'dberg's theorem is useful in the above discussions, it cannot be used to prove the respective inequalities $\rho(f) \geq \rho(A) \geq \rho(B)$ and $\rho_{\log }(f) \geq$ $\rho_{\log }(A) \geq \rho_{\log }(B)$ in Theorems 1 and 3 .

\section{Separation of Zeros}

We recall a few concepts from [20, Ch. V]. To shorten the notation, for $q>0$, let $\ell^{q}$ denote the standard sequence space consisting of sequences $\left\{w_{n}\right\}$ in $\mathbb{C}$ satisfying $\sum_{n}\left|w_{n}\right|^{q}<\infty$. We say that an infinite sequence $\left\{z_{n}\right\}$ in $\mathbb{C} \backslash\{0\}$ with no finite limit points has a finite exponent of convergence $\lambda>0$ if $\left\{1 /\left|z_{n}\right|\right\} \in \ell^{\lambda+\varepsilon} \backslash \ell^{\lambda-\varepsilon}$ for any $\varepsilon \in(0, \lambda)$, while $\lambda=0$ if $\left\{1 /\left|z_{n}\right|\right\} \in \ell^{\varepsilon}$ for any $\varepsilon>0$. The genus of $\left\{z_{n}\right\}$ is the unique integer $p \geq 0$ satisfying $\left\{1 /\left|z_{n}\right|\right\} \in \ell^{p+1} \backslash \ell^{p}$. If $\lambda \notin \mathbb{N} \cup\{0\}$, then $p=\lfloor\lambda\rfloor(=$ the integer part of $\lambda$ ), while if $\lambda \in \mathbb{N} \cup\{0\}$, then either $p=\lambda$ or $p=\lambda-1$. In all cases, $p \leq \lambda$. The Weierstrass convergence factors are

$$
e_{0}(z)=1 \text { and } e_{k}(z)=\exp \left(\sum_{j=1}^{k} \frac{z^{j}}{j}\right)
$$


where $k \in \mathbb{N}$. If $\left\{z_{n}\right\}$ has finite genus $p \geq 0$, then the canonical product

$$
f(z)=\prod_{n=1}^{\infty}\left(1-\frac{z}{z_{n}}\right) e_{p}\left(\frac{z}{z_{n}}\right)
$$

converges uniformly on compact subsets of $\mathbb{C}$, and hence represents an entire function having zeros precisely at the points $z_{n}$. We have $p \leq \lambda \leq \rho(f)$.

Our first observation in this section is that, even though the double inequalities $\rho(f) \geq \rho(A) \geq \rho(B)$ in Theorem 1 occur for solutions of (1.2), they do not always hold, as many examples show, including Examples 2 and 8.

Example 8 Let $\left\{z_{n}\right\}$ be the sequence defined by $z_{2 n-1}=2^{n}$ and $z_{2 n}=2^{n}+\varepsilon_{n}$, where $\left\{\varepsilon_{n}\right\}$ is any fixed sequence satisfying

$$
0<\varepsilon_{n}<\exp \left(-\exp \left(2^{n}\right)\right), \quad n \geq 1
$$

Thus the sequence $\left\{z_{n}\right\}$ has non-zero distinct points, and its exponent of convergence is equal to zero. Then the canonical product

$$
f(z)=\prod_{n=1}^{\infty}\left(1-\frac{z}{z_{n}}\right)
$$

is an entire function of order zero. Moreover,

$$
\begin{aligned}
f^{\prime}\left(z_{k}\right) & =-\frac{1}{z_{k}} \prod_{n \neq k}\left(1-\frac{z_{k}}{z_{n}}\right), \\
f^{\prime \prime}\left(z_{k}\right) & =\frac{2}{z_{k}} \sum_{m \neq k} \frac{1}{z_{m}} \prod_{j \neq k, m}\left(1-\frac{z_{k}}{z_{j}}\right),
\end{aligned}
$$

so that

$$
\sigma_{k}=-\frac{f^{\prime \prime}\left(z_{k}\right)}{f^{\prime}\left(z_{k}\right)}=2 \sum_{n \neq k} \frac{1}{z_{n}-z_{k}} .
$$

Then the reasoning in the proof of $[2$, Cor. 1] shows that

$$
\left|\sigma_{2 k-1}\right| \geq \exp \left(\exp \left(\left|z_{2 k-1}\right|\right)\right)+O(1) \text {. }
$$

Thus no finite order $A(z)$ can satisfy (3.1), even though $\rho(f)=0$. As before, we set $B(z)$ to be the function in (3.3), and then $\rho(B)=\infty$.

Obviously, many zeros of the function $f$ in (4.2) are close together. As stated in Sect. 1, the separation of zeros of the indicated solutions play a key role in the proofs 
of Theorems 1 and 3. For illustrative purposes, we discuss some examples regarding uniformly $q$-separated sequences (defined below).

Following an analogous definition in the unit disc [7], we say that a sequence $\left\{z_{n}\right\}$ of finite genus $p$ is uniformly $q$-separated for $q \geq 0$ provided that there exists a constant $C>0$ such that

$$
\inf _{k \in \mathbb{N}}\left\{e^{C\left|z_{k}\right|^{q}} \prod_{n \neq k}\left|1-\frac{z_{k}}{z_{n}}\right|\left|e_{p}\left(\frac{z_{k}}{z_{n}}\right)\right|\right\}>0 .
$$

An elementary differentiation of (4.1) followed by a substitution $z=z_{k}$ yields

$$
\begin{aligned}
f^{\prime}(z) & =-\sum_{j=1}^{\infty} \frac{z^{p}}{z_{j}^{p+1}} e_{p}\left(\frac{z}{z_{j}}\right) \prod_{n \neq j}\left(1-\frac{z}{z_{n}}\right) e_{p}\left(\frac{z}{z_{n}}\right), \\
f^{\prime}\left(z_{k}\right) & =-\frac{e_{p}(1)}{z_{k}} \prod_{n \neq k}\left(1-\frac{z_{k}}{z_{n}}\right) e_{p}\left(\frac{z_{k}}{z_{n}}\right),
\end{aligned}
$$

so that we may write (4.5) equivalently as

$$
\inf _{k \in \mathbb{N}}\left\{\left|z_{k}\right| e^{C\left|z_{k}\right|^{q}}\left|f^{\prime}\left(z_{k}\right)\right|\right\}>0 \text {. }
$$

The definition of a $q$-separated sequence in [13] assumes that $C=1$ in (4.7).

Example 9 We prove that the zeros of the function $f$ in (4.2) are not uniformly $q$ separated for any $q \geq 0$. Let $q \geq 0$, and set $k=2 n-1$. We have

$$
\begin{aligned}
\left|z_{k}\right|\left|f^{\prime}\left(z_{k}\right)\right| & =\left|1-\frac{z_{k}}{z_{k+1}}\right| \prod_{j \neq k, k+1}\left|1-\frac{z_{k}}{z_{j}}\right| \\
& =\frac{z_{k+1}-z_{k}}{z_{k+1}} \cdot \exp \left(\sum_{j \neq k, k+1} \log \left|1-\frac{z_{k}}{z_{j}}\right|\right) \\
& \leq \frac{\varepsilon_{n}}{2^{n}+\varepsilon_{n}} \cdot \exp \left(\sum_{j \neq k, k+1} \log \left(1+\left|\frac{z_{k}}{z_{j}}\right|\right)\right) \\
& \leq \frac{\varepsilon_{n}}{2^{n}} \cdot \exp \left(\sum_{j=1}^{\infty}\left|\frac{z_{k}}{z_{j}}\right|\right) \leq \frac{\varepsilon_{n}}{2^{n}} \cdot \exp \left(K\left|z_{k}\right|\right)
\end{aligned}
$$

for some constant $K>0$ independent of $k$. Thus, for every $C>0$,

$$
\left|z_{k}\right| e^{C\left|z_{k}\right|^{q}}\left|f^{\prime}\left(z_{k}\right)\right| \leq \exp \left(C 2^{n q}+K 2^{n}-n \log 2-\exp \left(2^{n}\right)\right) \rightarrow 0
$$

as $n \rightarrow \infty$ (or equivalently as $k \rightarrow \infty$ ). 
Remark 2 In Example 8, we showed that the function $f$ in (4.2) could not satisfy the conclusion in Theorem 1, and above we showed that the zeros of this function are not uniformly $q$-separated for any $q \geq 0$. In contrast, the zeros of the indicated solution $f$ in Theorem 1 (the Lindelöf function $L_{\rho}$ ) are uniformly $q$-separated for every $q>\rho(f)$, see Sect. 6 .

For completeness, we construct an example of a uniformly $q$-separated sequence for $q>0$ and $\lambda=0$.

Example 10 The sequence $\left\{z_{n}\right\}$ given by $z_{n}=2^{n}$ is uniformly 0 -separated and has zero exponent of convergence [13, p. 299]. For a fixed $q>0$, choose $\gamma_{n} \in$ $\left[\min \left\{1 / 2,2^{n} \exp \left(-2^{n q}\right)\right\}, 1\right)$, and define $w_{n}=z_{n}+\gamma_{n}$. Then $\left\{w_{n}\right\}$ is also uniformly 0 -separated and has zero exponent of convergence. Let $\left\{\zeta_{n}\right\}$ denote the union sequence $\left\{z_{n}\right\} \cup\left\{w_{n}\right\}$. Construct canonical products $P_{1}(z)$ and $P_{2}(z)$ with zero sequences $\left\{z_{n}\right\}$ and $\left\{w_{n}\right\}$, respectively, and define $P(z)=P_{1}(z) P_{2}(z)$. Then a calculation in the spirit of Example 9 , with $P$ in place of $f$, shows that $\left\{\zeta_{n}\right\}$ is uniformly $q$-separated. The details are omitted.

\section{Preparations for the Proof of Theorem 1}

The following auxiliary result is a modification of [13, Cor. 3.3] that is needed to find an entire $A(z)$ satisfying the interpolation problem (3.1) in our proof of Theorem 1. As we see, the growth of such an $A(z)$ depends heavily on the uniform $q$-separation of the zeros of $f$. This needs to be taken into account when proving the inequality $\rho(f) \geq \rho(A)$.

Lemma 3 Suppose that $\left\{z_{n}\right\}$ is an infinite sequence of non-zero points in $\mathbb{C}$ with finite exponent of convergence $\lambda$, and that $\left\{z_{n}\right\}$ is uniformly $q$-separated for some $q \geq 0$ (and $C>0$ ). Let $\left\{\sigma_{n}\right\}$ be an infinite sequence of points in $\mathbb{C}$, not necessarily distinct, and let $h:[0, \infty) \rightarrow[1, \infty)$ be a continuous and non-decreasing function such that $\left|\sigma_{n}\right| \leq h\left(\left|z_{n}\right|\right)$ for $n \in \mathbb{N}$.

Then there exists an entire function $A(z) \not \equiv 0$ such that

$$
A\left(z_{n}\right)=\sigma_{n}, \quad n \in \mathbb{N},
$$

and, for any given $\alpha>1$,

$$
\rho(A) \leq \max \left\{\lambda, \limsup _{r \rightarrow \infty} \frac{\log I(\alpha r)}{\log r}\right\},
$$

where $I(x)=\max _{e \leq t \leq x} \frac{\log \left(h(t) e^{C t^{q}}\right)}{\log t}$ is a non-decreasing function for $x \geq e$.

Proof Without loss of generality, we may suppose that $\left\{z_{n}\right\}$ is ordered according to increasing moduli. Moreover, $I(x)$ is well defined and non-decreasing by continuity. Let $P(z)$ be the canonical product of genus $p \leq \lambda$ having $\left\{z_{n}\right\}$ as its zero sequence. By uniform $q$-separation, the points $z_{n}$ are simple, and hence $1 / P^{\prime}\left(z_{n}\right) \in \mathbb{C}$ for any $n$. 
(a) Suppose that $\sigma_{n} \neq 0$ for all $n$. Let $H(z)$ denote the Mittag-Leffler series in [13, Eq. (3.1)], i.e.,

$$
H(z)=\sum_{n=1}^{\infty} \frac{c_{n}}{z-z_{n}}\left(\frac{z}{z_{n}}\right)^{q_{n}}
$$

where $c_{n}=\sigma_{n} / P^{\prime}\left(z_{n}\right)$ and $\left\{q_{n}\right\}$ is a sequence such that each $q_{n} \geq 0$ is the smallest integer satisfying

$$
q_{n} \geq \max \left\{\alpha\left(\frac{\log \left|c_{n}\right|}{\log \left|z_{n}\right|}+p\right), \frac{\log \frac{\left|c_{n}\right|}{n}}{\log \left|z_{n}\right|}+p+1\right\}
$$

for $\left|z_{n}\right|>e$, and set $q_{n}=0$ otherwise. Then $H(z)$ is meromorphic in $\mathbb{C}$ and $A=P H$ is an entire function that satisfies (5.1). Hence it suffices to prove (5.2).

By the assumptions, there exists a constant $C_{1}>0$ such that

$$
\left|c_{n}\right| \leq C_{1}\left|z_{n}\right| h\left(\left|z_{n}\right|\right) e^{C\left|z_{n}\right|^{q}}, \quad n \in \mathbb{N}
$$

Thus

$$
\frac{\log \left|c_{n}\right|}{\log \left|z_{n}\right|} \leq \frac{\log \left(h\left(\left|z_{n}\right|\right) e^{C\left|z_{n}\right|^{q}}\right)}{\log \left|z_{n}\right|}+C_{2}, \quad\left|z_{n}\right| \geq e,
$$

where $C_{2}>0$ is a constant. Set

$$
g(t)=\frac{\log \left(h(t) e^{C t^{q}}\right)}{\log t}+C_{2}, \quad t \geq e,
$$

so that the inequality

$$
\frac{\log \left|c_{n}\right|}{\log \left|z_{n}\right|} \leq g\left(\left|z_{n}\right|\right)
$$

holds for every $n$ such that $\left|z_{n}\right| \geq e$. As in the proof of [13, Cor. 3.3], we would like to apply [13, Thm. 3.1] next, but the monotonicity of $g(t)$ is not known, in particular when $0 \leq q<1$. Thus we replace $g(t)$ with

$$
G(t)=\max _{e \leq x \leq t} g(x)
$$

which is a non-decreasing function by continuity. Now [13, Thm. 3.1] gives us

$$
\rho(H) \leq \max \left\{\lambda, \limsup _{r \rightarrow \infty} \frac{\log G(\alpha r)}{\log r}\right\}
$$


Indeed, it is apparent from the proof of [13, Thm. 3.1] that the finitely many indices $n$ for which $\left|z_{n}\right|<e$ have no affect on this conclusion. The assertion (5.2) then follows from $\rho(A) \leq \max \{\rho(P), \rho(H)\}$.

(b) Suppose that $\sigma_{n}=0$ for at least one $n$. If $\sigma_{n}=0$ for all $n$, we may choose $A(z)=$ $P(z)$, in which case (5.1) and (5.2) clearly hold. Hence we may suppose that $\sigma_{n}=0$ for at least one index $n$ but not for all $n$. Let $\left\{s_{n}\right\}$ denote the subsequence of $\left\{\sigma_{n}\right\}$ consisting of all the non-zero points. Let $\left\{z_{n}\right\}=\left\{\zeta_{n}\right\} \cup\left\{\xi_{n}\right\}$ be a partition of the sequence $\left\{z_{n}\right\}$ such that each $\zeta_{n}$ corresponds to $s_{n}$. In other words, the interpolation problem (5.1) is transformed into finding an entire function $A(z)$ such that

$$
A\left(\zeta_{n}\right)=s_{n} \text { and } A\left(\xi_{n}\right)=0
$$

We factorize the canonical product $P(z)$ as $P(z)=R(z) S(z)$, where $R\left(\zeta_{n}\right)=0$ and $S\left(\xi_{n}\right)=0$. Supposing that $\left\{s_{n}\right\}$ is an infinite sequence, let $H(z)$ denote the Mittag-Leffler series (5.3), where $c_{n}=s_{n} / P^{\prime}\left(\zeta_{n}\right) \neq 0$. Then $A=P H$ is entire and satisfies (5.5). The growth condition (5.2) is then proved just as in Part (a). If $\left\{s_{n}\right\}$ is a finite sequence, then for $H(z)$ we choose a finite Mittag-Leffler series. Once again $A(z)=P(z) H(z)$ is entire and satisfies (5.5) along with $\rho(A)=\rho(P)=\lambda$.

Remark 4 (a) The proof of Lemma 3 differs from that of [13, Cor. 3.3] in two ways: The possibility that $\sigma_{n}=0$ for some $n$ is now included, and the growth of the majorant function $g(t)$ has been considered in more detail.

(b) Lemma 3 will be applied to the target sequence $\sigma_{k}=-f^{\prime \prime}\left(z_{k}\right) / f^{\prime}\left(z_{k}\right)$ in (3.1), where $f$ is a canonical product with genus $p \geq 0$. A very similar target sequence appears in the proof of [2, Thm. 1]. We have the representation

$$
\sigma_{k}=-\frac{2 p}{z_{k}}+2 \sum_{n \neq k}\left(\frac{z_{k}}{z_{n}}\right)^{p} \frac{1}{z_{n}-z_{k}}
$$

which was proved for $p=0$ in Example 8. For $p \geq 1$, we have the first-order derivatives (4.6). A second differentiation yields

$$
\begin{aligned}
f^{\prime \prime}\left(z_{k}\right)= & -\frac{2 p}{z_{k}^{2}} e_{p}(1) \prod_{j \neq k}\left(1-\frac{z_{k}}{z_{j}}\right) e_{p}\left(\frac{z_{k}}{z_{j}}\right) \\
& +\frac{2}{z_{k}} e_{p}(1) \sum_{n \neq k} \frac{z_{k}^{p}}{z_{n}^{p+1}} e_{p}\left(\frac{z_{k}}{z_{n}}\right) \prod_{j \neq n, k}\left(1-\frac{z_{k}}{z_{j}}\right) e_{p}\left(\frac{z_{k}}{z_{j}}\right),
\end{aligned}
$$

which together with (4.6) implies (5.6). 
(c) Let $f$ be an entire function having simple zeros at the points $a_{n}$, and let $\left\{b_{n}\right\}$ be a target sequence. Then the Lagrange interpolation series

$$
L(z)=\sum_{n=1}^{\infty} \frac{b_{n} f(z)}{f^{\prime}\left(a_{n}\right)\left(z-a_{n}\right)}
$$

is an entire solution to the interpolation problem $L\left(a_{n}\right)=b_{n}$, provided that $L(z)$ converges uniformly on compact subsets of $\mathbb{C}$, see [16, p. 195]. In our case, however, the target sequence is unbounded, and the zero-sequence $\left\{a_{n}\right\}$ is uniformly $q$-separated. The latter means in general that $\left|f^{\prime}\left(a_{n}\right)\right|$ can tend to zero exponentially as $n \rightarrow \infty$. Thus it seems unlikely that the Lagrange interpolation series could be used in proving Theorem 1, and the use of Lemma 3 instead seems to be justified.

\section{Proof of Theorem 1}

The solution $f$ will be the Lindelöf function $L_{\rho}$ of order $\rho>1 / 2$, which is a canonical product with simple zeros precisely at the points $z_{n}=-n^{1 / \rho}, n \geq 1$, on the negative real axis. We may write

$$
L_{\rho}(z)=\prod_{n=1}^{\infty}\left(1+\frac{z}{n^{\alpha}}\right) e_{p}\left(-\frac{z}{n^{\alpha}}\right)
$$

where $\alpha=1 / \rho$ and $p=\lfloor\rho\rfloor\left(=\right.$ integer part of $\rho$ ) is the genus of $L_{\rho}$. It is known (see $[5$, p. 294] or $[17$, p. 54]) that

$$
\delta_{N}\left(0, L_{\rho}\right)= \begin{cases}1-\frac{|\sin (\pi \rho)|}{q+|\sin (\pi \rho)|}, & q<\rho \leq q+\frac{1}{2}, \\ 1-\frac{|\sin (\pi \rho)|}{q+1}, & q+\frac{1}{2}<\rho \leq q+1,\end{cases}
$$

where $q \geq 0$ is an integer. It follows that $\delta_{N}\left(0, L_{\rho}\right)>0$ for every $\rho>1 / 2$. Since entire functions of order $\leq 1 / 2$ have no finite Nevanlinna deficient values, the Lindelöf functions illustrate the sharpness of this inequality.

Lemma 5 For $k, n \in \mathbb{N}, k>n$, and $\alpha>0$, we have

$$
\begin{array}{ll}
\alpha(k-n) k^{\alpha-1} \leq k^{\alpha}-n^{\alpha} \leq \alpha(k-n) n^{\alpha-1}, & 0<\alpha \leq 1, \\
\alpha(k-n) n^{\alpha-1} \leq k^{\alpha}-n^{\alpha} \leq \alpha(k-n) k^{\alpha-1}, & 1 \leq \alpha<\infty .
\end{array}
$$

In particular, for every $\alpha>0$, we have $k^{\alpha}-(k-1)^{\alpha} \sim \alpha k^{\alpha-1}$ as $k \rightarrow \infty$.

The crux of the proof of Lemma 5 is a simple identity

$$
k^{\alpha}-n^{\alpha}=\alpha \int_{n}^{k} x^{\alpha-1} d x
$$


where the integrand $x^{\alpha-1}$ is decreasing for $0<\alpha<1$ and non-decreasing for $\alpha \geq 1$. We omit the details.

Lemma 6 Let $L_{\rho}$ be the Lindelöf function of order $\rho>1 / 2$ with zeros $z_{k}=-k^{1 / \rho}$ of genus $p=\lfloor\rho\rfloor$. Then the (target) sequence $\sigma_{k}=-L_{\rho}^{\prime \prime}\left(z_{k}\right) / L_{\rho}^{\prime}\left(z_{k}\right)$ can be written as

$$
\sigma_{k}=\frac{2 p}{k^{\alpha}}+2 \sum_{n \neq k}\left(\frac{k}{n}\right)^{\alpha p} \frac{1}{k^{\alpha}-n^{\alpha}}
$$

where $\alpha=1 / \rho$. Moreover,

$$
\left|\sigma_{k}\right|= \begin{cases}O(\log k), & p=0 \\ O\left(k^{\alpha(p-1)+1} \log k\right), & p \geq 1\end{cases}
$$

Proof The representation (6.4) follows immediately from (5.6). We estimate the growth of $\left|\sigma_{k}\right|$ in two steps.

(1) Suppose that $p=0$. Then $\rho \in(1 / 2,1)$, i.e., $\alpha \in(1,2)$. Using $(6.4)$, we get

$$
\left|\sigma_{k}\right| / 2 \leq \sum_{n \leq k-1} \frac{1}{k^{\alpha}-n^{\alpha}}+\sum_{n \geq k+1} \frac{1}{n^{\alpha}-k^{\alpha}}=: S_{1}(k)+S_{2}(k),
$$

where $S_{1}(1)=0$. Suppose that $k \geq 2$. Since $x \mapsto 1 /\left(k^{\alpha}-x^{\alpha}\right)$ is an increasing function for $x \in[1, k-1]$, the left endpoint rule and (6.3) give

$$
\begin{aligned}
S_{1}(k) & \leq \int_{1}^{k-1} \frac{d x}{k^{\alpha}-x^{\alpha}}+\frac{1}{k^{\alpha}-(k-1)^{\alpha}} \\
& \leq \int_{1}^{k-1} \frac{\alpha x^{\alpha-1} d x}{k^{\alpha}-x^{\alpha}}+\frac{1}{\alpha(k-1)^{\alpha-1}}=O(\log k) .
\end{aligned}
$$

Analogously, $x \mapsto 1 /\left(x^{\alpha}-k^{\alpha}\right)$ is a decreasing function for $x \geq k+1$, so the right endpoint rule and (6.3) give

$$
\begin{aligned}
S_{2}(k) & \leq \int_{k+1}^{\infty} \frac{d x}{x^{\alpha}-k^{\alpha}}+\frac{1}{(k+1)^{\alpha}-k^{\alpha}} \\
& \leq \frac{1}{k^{\alpha-1}} \int_{(k+1) / k}^{\infty} \frac{d t}{t^{\alpha}-1}+\frac{1}{\alpha k^{\alpha-1}}
\end{aligned}
$$

where we made the change of variable $t=x / k$. It is clear that

$$
\int_{2}^{\infty} \frac{d t}{t^{\alpha}-1} \leq \frac{2^{\alpha}}{2^{\alpha}-1} \int_{2}^{\infty} \frac{d t}{t^{\alpha}}=\frac{2}{(\alpha-1)\left(2^{\alpha}-1\right)}
$$


Since $\left(t^{\alpha}-1\right) /\left(t^{2}-1\right) \rightarrow \alpha / 2$ as $t \rightarrow 1^{+}$, there exists, by continuity, a constant $\beta \in(0, \alpha / 2)$ such that $t^{\alpha}-1 \geq \beta\left(t^{2}-1\right)$ for all $t \in[1,2]$. Therefore

$$
\int_{(k+1) / k}^{2} \frac{d t}{t^{\alpha}-1} \leq \frac{1}{\beta} \int_{(k+1) / k}^{2} \frac{d t}{t^{2}-1}=\frac{1}{2 \beta} \log \left(\frac{2 k+1}{3}\right) \leq \frac{1}{2 \beta} \log k .
$$

This yields $S_{2}(k)=O\left(k^{1-\alpha} \log k\right)=o(1)$, and, a fortiori, $\left|\sigma_{k}\right|=O(\log k)$.

(2) Suppose that $p \geq 1$. Then $\rho \in[1, \infty)$, i.e., $\alpha \in(0,1]$. Using (6.4), we get

$$
\begin{aligned}
\left|\sigma_{k}\right| / 2-\frac{p}{k^{\alpha}} & \leq \sum_{n \leq k-1}\left(\frac{k}{n}\right)^{\alpha p} \frac{1}{k^{\alpha}-n^{\alpha}}+\sum_{n \geq k+1}\left(\frac{k}{n}\right)^{\alpha p} \frac{1}{n^{\alpha}-k^{\alpha}} \\
& =: T_{1}(k)+T_{2}(k),
\end{aligned}
$$

where $T_{1}(1)=0$. Now $p \leq \rho<p+1$, so that

$$
\alpha p=\frac{p}{\rho} \in\left(\frac{p}{p+1}, 1\right] \text { and } \alpha(p+1)=\frac{p+1}{\rho} \in\left(1, \frac{p+1}{p}\right] \text {. }
$$

Suppose that $k \geq 2$. Then (6.2) yields

$$
\begin{aligned}
T_{1}(k) & \leq \sum_{n \leq k-1} \frac{k^{\alpha p}}{k^{\alpha}-n^{\alpha}} \leq \int_{1}^{k-1} \frac{k^{\alpha p}}{k^{\alpha}-x^{\alpha}} d x+\frac{k^{\alpha p}}{k^{\alpha}-(k-1)^{\alpha}} \\
& \leq \frac{k^{\alpha(p-1)+1}}{\alpha}\left(\int_{1}^{k-1} \frac{\alpha x^{\alpha-1}}{k^{\alpha}-x^{\alpha}} d x+1\right) \leq \frac{k^{\alpha(p-1)+1}}{\alpha}\left(\log \frac{k}{\alpha}+1\right)
\end{aligned}
$$

or $T_{1}(k)=O\left(k^{\alpha(p-1)+1} \log k\right)$. The function $x \mapsto\left(x^{\alpha p}\left(x^{\alpha}-k^{\alpha}\right)\right)^{-1}$ is strictly decreasing for $x \geq k+1$, so that

$$
\begin{aligned}
T_{2}(k) & \leq \int_{k+1}^{\infty} \frac{k^{\alpha p}}{x^{\alpha p}\left(x^{\alpha}-k^{\alpha}\right)} d x+\frac{1}{(k+1)^{\alpha}-k^{\alpha}} \\
& \leq k^{1-\alpha} \int_{(k+1) / k}^{\infty} \frac{d t}{t^{\alpha p}\left(t^{\alpha}-1\right)}+\frac{(k+1)^{1-\alpha}}{\alpha}
\end{aligned}
$$

where we made the change of variable $t=x / k$. It is clear that

$$
\int_{2}^{\infty} \frac{d t}{t^{\alpha p}\left(t^{\alpha}-1\right)} \leq \frac{2^{\alpha}}{2^{\alpha}-1} \int_{2}^{\infty} \frac{d t}{t^{\alpha(p+1)}}=C(\alpha, p)<\infty
$$

Analogously as in Part (1) of the proof, we obtain

$$
\int_{(k+1) / k}^{2} \frac{d t}{t^{\alpha p}\left(t^{\alpha}-1\right)} \leq \int_{(k+1) / k}^{2} \frac{d t}{t^{\alpha}-1} \leq \frac{1}{2 \beta} \log k
$$


for some $\beta \in(0, \alpha / 2)$. This yields $T_{2}(k)=O\left(k^{1-\alpha} \log k\right)$. The desired estimate for $\left|\sigma_{k}\right|$ follows from the estimates for $T_{1}(k)$ and $T_{2}(k)$.

The estimates in the previous proof seem to have some flexibility. Hence the result is unlikely to be sharp, but it is nevertheless more than enough for our use.

Lemma 7 Let $L_{\rho}$ be the Lindelöf function of order $\rho \in(1 / 2, \infty)$ with zeros $z_{k}=$ $-k^{1 / \rho}$ of genus $p=\lfloor\rho\rfloor$. Then there exists a constant $C>0$ such that

$$
\inf _{k}\left\{\left|z_{k}\right| e^{C\left|z_{k}\right|^{\rho} \log \left|z_{k}\right|^{\rho}}\left|L_{\rho}^{\prime}\left(z_{k}\right)\right|\right\} \geq 1 .
$$

In particular, the zero sequence of $L_{\rho}$ is uniformly $q$-separated for every $q>\rho$.

Proof Set $\alpha=1 / \rho$ for brevity. As the final conclusion is trivial, it suffices to prove (6.6). We do this in two steps.

(1) Suppose that $\rho \in(1 / 2,1)$, i.e., $\alpha \in(1,2)$. By appealing to (4.3) and (6.1), we have

$$
\left|z_{k}\right|\left|L_{\rho}^{\prime}\left(z_{k}\right)\right|=\prod_{n \leq k-1}\left(\frac{k^{\alpha}}{n^{\alpha}}-1\right) \prod_{n \geq k+1}\left(1-\frac{k^{\alpha}}{n^{\alpha}}\right)=: P_{1}(k) P_{2}(k),
$$

where $P_{1}(1)=1$. The products $P_{1}(k)$ and $P_{2}(k)$ converge for any finite $k$, so it suffices to estimate them for arbitrarily large values of $k$. We will make use of (6.3) in these estimates.

We estimate $P_{1}(k)$ from below by

$$
\begin{aligned}
P_{1}(k)^{-1} & =\prod_{n=1}^{k-1} \frac{n^{\alpha}}{k^{\alpha}-n^{\alpha}}=\exp \left(\sum_{n=1}^{k-1} \log \frac{n^{\alpha}}{k^{\alpha}-n^{\alpha}}\right) \leq \exp \left(\sum_{n=1}^{k-1} \log \frac{n}{\alpha(k-n)}\right) \\
& =\exp \left((k-1) \log \frac{1}{\alpha}+\sum_{n=1}^{k-1} \log \frac{n}{k-n}\right)=\exp (-(k-1) \log \alpha) \leq 1,
\end{aligned}
$$

where $k \geq 2$. When estimating $P_{2}(k)$ from below, we apply the right endpoint rule to the decreasing function $x \mapsto \log \left(1+k^{\alpha} /\left(x^{\alpha}-k^{\alpha}\right)\right)$ on $[k+1, \infty)$, and make use of Part (1) in the proof of Lemma 6. We conclude that

$$
\begin{aligned}
P_{2}(k)^{-1} & =\prod_{n \geq k+1} \frac{n^{\alpha}}{n^{\alpha}-k^{\alpha}}=\exp \left(\sum_{n \geq k+1} \log \left(1+\frac{k^{\alpha}}{n^{\alpha}-k^{\alpha}}\right)\right) \\
& \leq \exp \left(\int_{k+1}^{\infty} \log \left(1+\frac{k^{\alpha}}{x^{\alpha}-k^{\alpha}}\right) d x+\log \frac{(k+1)^{\alpha}}{(k+1)^{\alpha}-k^{\alpha}}\right) \\
& \leq \exp \left(\int_{k+1}^{\infty} \frac{k^{\alpha}}{x^{\alpha}-k^{\alpha}} d x+\log \frac{(k+1)^{\alpha} k}{\alpha k^{\alpha}}\right) \\
& \leq \exp \left(k \int_{(k+1) / k}^{\infty} \frac{d t}{t^{\alpha}-1}+\log \frac{2^{\alpha} k}{\alpha}\right) \leq \exp \left(C_{1} k \log k\right)
\end{aligned}
$$


for some $C_{1}=C_{1}(\alpha)>0$ and for all $k \geq 2$.

Finally, we combine the estimates for $P_{1}(k)$ and $P_{2}(k)$, and obtain

$$
\left|z_{k}\right| e^{C\left|z_{k}\right|^{\rho} \log \left|z_{k}\right|^{\rho}}\left|L_{\rho}^{\prime}\left(z_{k}\right)\right|=e^{C k \log k} P_{1}(k) P_{2}(k) \geq 1, \quad k \geq 2,
$$

where $C$ is any constant satisfying $C \geq C_{1}$. This completes the proof of (6.6) in the case $\rho \in(1 / 2,1)$.

(2) Suppose that $\rho \in[1, \infty)$, i.e., $\alpha \in(0,1]$. By appealing to (4.6) and (6.1), we have

$$
\begin{aligned}
\frac{\left|z_{k}\right|\left|L_{\rho}^{\prime}\left(z_{k}\right)\right|}{e_{p}(1)} & =\prod_{n \leq k-1}\left(\frac{k^{\alpha}}{n^{\alpha}}-1\right) e_{p}\left(\frac{k^{\alpha}}{n^{\alpha}}\right) \prod_{n \geq k+1}\left(1-\frac{k^{\alpha}}{n^{\alpha}}\right) e_{p}\left(\frac{k^{\alpha}}{n^{\alpha}}\right) \\
& =: Q_{1}(k) Q_{2}(k),
\end{aligned}
$$

where $Q_{1}(1)=1$. The products $Q_{1}(k)$ and $Q_{2}(k)$ converge for any finite $k$, so it suffices to estimate them for arbitrarily large values of $k$. We make use of (6.2) and (6.3) in these estimates.

A trivial elimination of the Weierstrass convergence factor allows us to argue analogously as in estimating $P_{1}(k)$ in Part (1) of the proof. In addition, we make use of the inequality $n^{n} \leq e^{n} n$ !, which is valid for all $n \in \mathbb{N}$. We have

$$
\begin{aligned}
Q_{1}(k)^{-1} & =\prod_{n=1}^{k-1} \frac{n^{\alpha}}{k^{\alpha}-n^{\alpha}} e_{p}\left(\frac{k^{\alpha}}{n^{\alpha}}\right)^{-1} \leq \exp \left(\sum_{n=1}^{k-1} \log \frac{n^{\alpha}}{k^{\alpha}-n^{\alpha}}\right) \\
& \leq \exp \left(\sum_{n=1}^{k-1} \log \frac{k n^{\alpha}}{\alpha(k-n) k^{\alpha}}\right) \\
& =\exp \left((k-1) \log \frac{1}{\alpha}+(1-\alpha) \log \frac{k^{k-1}}{(k-1) !}\right) \leq \exp \left(D_{1} k\right)
\end{aligned}
$$

where $k \geq 2$ and $D_{1}=\log (1 / \alpha)+1-\alpha \geq 0$ for $0<\alpha \leq 1$.

Before estimating $Q_{2}(k)$, we make the preliminary manipulations

$$
\begin{aligned}
Q_{2}(k)^{-1} & =\prod_{n \geq k+1} \frac{n^{\alpha}}{n^{\alpha}-k^{\alpha}} \exp \left(-\sum_{j=1}^{p} \frac{1}{j}\left(\frac{k^{\alpha}}{n^{\alpha}}\right)^{j}\right) \\
& =\exp \left(\sum_{n=k+1}^{\infty}\left(\log \left(\frac{1}{1-k^{\alpha} / n^{\alpha}}\right)-\sum_{j=1}^{p} \frac{1}{j}\left(\frac{k^{\alpha}}{n^{\alpha}}\right)^{j}\right)\right) \\
& =\exp \left(\sum_{n=k+1}^{\infty} \sum_{j=p+1}^{\infty} \frac{1}{j}\left(\frac{k^{\alpha}}{n^{\alpha}}\right)^{j}\right) .
\end{aligned}
$$


A simple reasoning based on geometric series yields

$$
\begin{aligned}
Q_{2}(k)^{-1} & \leq \exp \left(\sum_{n=k+1}^{\infty}\left(\frac{k^{\alpha}}{n^{\alpha}}\right)^{p+1} \sum_{j=0}^{\infty}\left(\frac{k^{\alpha}}{n^{\alpha}}\right)^{j}\right) \\
& =\exp \left(\sum_{n=k+1}^{\infty}\left(\frac{k}{n}\right)^{\alpha(p+1)} \frac{n^{\alpha}}{n^{\alpha}-k^{\alpha}}\right) \\
& =\exp \left(k^{\alpha} \sum_{n=k+1}^{\infty}\left(\frac{k}{n}\right)^{\alpha p} \frac{1}{n^{\alpha}-k^{\alpha}}\right)
\end{aligned}
$$

The estimate for $T_{2}(k)$ in the proof of Lemma 6 then yields $Q_{2}(k)^{-1} \leq$ $\exp \left(D_{2} k \log k\right)$ for some $D_{2}=D_{2}(\alpha)>0$ and for all $k \geq 2$.

Finally, we combine the estimates for $Q_{1}(k)$ and $Q_{2}(k)$, and obtain

$$
\left|z_{k}\right| e^{C\left|z_{k}\right|^{\rho} \log \left|z_{k}\right|^{\rho}}\left|L_{\rho}^{\prime}\left(z_{k}\right)\right|=e^{C k \log k} Q_{1}(k) Q_{2}(k) \geq 1,
$$

where $C$ is any constant satisfying $C>D_{1}+D_{2}$ and $k \geq 2$ is large enough. This completes the proof of (6.6) in the case $\rho \in[1, \infty)$.

Proof of Theorem 1 After these preparations, the actual proof of Theorem 1 is now easy. Let $f=L_{\rho}$ be the Lindelöf function of order $\rho \in(1 / 2, \infty)$, which has simple zeros and the required growth. In addition, the zeros of $f$ are uniformly $q$-separated for every $q>\rho$ by Lemma 7 . For $K \geq 1$, define $h:[0, \infty) \rightarrow[1, \infty)$ by

$$
h(t)= \begin{cases}K \log \left(t^{\rho}+e\right), & p=0 \\ K\left(t^{\rho(\alpha(p-1)+1)} \log \left(t^{\rho}+e\right)+1\right), & p \geq 1\end{cases}
$$

Then

$$
h\left(\left|z_{k}\right|\right)=h\left(k^{\alpha}\right)= \begin{cases}K \log (k+e), & p=0 \\ K\left(k^{\alpha(p-1)+1} \log (k+e)+1\right), & p \geq 1 .\end{cases}
$$

By Lemma 6, we may choose $K \geq 1$ so that $\left|\sigma_{k}\right| \leq h\left(\left|z_{k}\right|\right)$ for all $k$. Moreover, for all $p \geq 0$, we have $\log h(t)=O(\log t)$. Thus

$$
I(x):=\max _{e \leq t \leq x} \frac{\log \left(h(t) e^{C t^{q}}\right)}{\log t} \leq \max _{e \leq t \leq x} \log \left(h(t) e^{C t^{q}}\right)=C x^{q}+O(\log x),
$$

and we conclude by Lemma 3 that there exists an entire function $A(z) \not \equiv 0$ satisfying (3.1) such that

$$
\rho(A) \leq \max \{\rho(f), q\}
$$


The zero sequence $\left\{z_{n}\right\}$ of $f$ that determines $A$ in Lemma 3 is fixed, but it is also uniformly $q$-separated for any $q>\rho(f)$ by Lemma 7. Thus the estimate in (6.8) provided by Lemma 3 holds for any $q>\rho(f)$, and, a fortiori, $\rho(A) \leq \rho(f)$.

Finally, we define the entire coefficient $B(z)$ by (3.3), for which $\rho(B) \leq \rho(A)$ holds by the lemma on the logarithmic derivative. It remains to show that $B(z) \not \equiv 0$. Suppose on the contrary that $B(z) \equiv 0$, in which case (1.2) reduces to $f^{\prime \prime}+A(z) f^{\prime}=0$. A simple integration shows that

$$
f^{\prime}(z)=\exp \left(\int^{z} A(\zeta) d \zeta\right)
$$

Since $f$ (and hence $f^{\prime}$ ) is of finite order, it follows that $A(z)$ is a polynomial. This gives us $\rho(f)=\operatorname{deg}(A)+1 \geq 1$. Since $\rho(f) \in \mathbb{N}$, it is clear from the definition of the Lindelöf function that $p=\rho(f)$. However, according to the representation (4.6), the derivative $f^{\prime}$ should have a $p$-fold zero at the origin, which is a contradiction. This completes the proof.

\section{Preparations for the Proof of Theorem 3}

The separation of the zeros of the constructed solution $f$ of (1.2) plays a key role in the proof of Theorem 3. The zeros of $f$ are uniformly logarithmically $q$-separated (defined below) for every $q>\rho_{\log }(f)-1$.

For basic properties of entire (or, more generally, meromorphic) functions of finite logarithmic order, we refer to $[3,4]$. In particular, the logarithmic exponent of convergence of the zeros of an entire $f$ is given by

$$
\lambda_{\log }(f)=\limsup _{r \rightarrow \infty} \frac{\log n(r)}{\log \log r},
$$

where $n(r)$ denotes the number of zeros of $f$ in $|z|<r$, counting multiplicities. Similarly to the usual order, the functions $T(r, f)$ and $\log M(r, f)$ have the same logarithmic order $\rho_{\log }$, and $\rho_{\log }\left(f^{\prime}\right)=\rho_{\log }(f)$. Differing from the usual order, where $\lambda(f) \leq \rho(f)$, we have $\rho_{\log }(f)=\lambda \log (f)+1$. This reflects the fact that polynomials are of logarithmic order one, and they have only finitely many zeros.

We require a new concept on point separation. We say that a sequence $\left\{z_{n}\right\}$ of finite genus $p$ is uniformly logarithmically $q$-separated for $q \geq 0$ provided that there exists a constant $C>0$ such that

$$
\inf _{k \in \mathbb{N}}\left\{e^{C\left(\log \left(1+\left|z_{k}\right|\right)\right)^{q}} \prod_{n \neq k}\left|1-\frac{z_{k}}{z_{n}}\right|\left|e_{p}\left(\frac{z_{k}}{z_{n}}\right)\right|\right\}>0 .
$$

If $f$ is given by (4.1), then using (4.6), we may write (7.1) equivalently as

$$
\inf _{k \in \mathbb{N}}\left\{\left|z_{k}\right| e^{C\left(\log \left(1+\left|z_{k}\right|\right)\right)^{q}}\left|f^{\prime}\left(z_{k}\right)\right|\right\}>0 .
$$


Certainly there exist sequences with zero exponent of convergence which are not uniformly logarithmically $q$-separated for any $q \geq 0$. Moreover, uniformly logarithmically 0 -separated sequences are uniformly 0 -separated sequences, and vice versa.

We will make use of this new separation concept for sequences of finite logarithmic exponent of convergence, in which case $p=0$ and the corresponding $f$ reduces to the form (4.2). The definition in (7.1) for general $p$ is given for possible applications in the future. Moreover, one could replace the polynomial and logarithmic weights by some monotonic function $\varphi: \mathbb{R}_{+} \rightarrow \mathbb{R}$, and discuss uniform $\varphi$-separation.

In the next example we will discuss the case $\lambda_{\log }=1$ and $q>0$.

Example 11 Let $z_{n}=2^{n}$ and $w_{n}=z_{n}+\varepsilon_{n}$, where $q>0$ and

$$
\varepsilon_{n}=\min \left\{1 / 2,2^{n} \exp \left(-(n \log 2)^{q}\right)\right\}, \quad n \geq 1 .
$$

Then $z_{n}<w_{n}<z_{n+1}, w_{n+1} \geq z_{n+1} \geq 3 w_{n} / 2$ and $w_{n} \leq z_{n}+1 / 2 \leq 4 z_{n} / 3$. A simple modification of the reasoning in Example 10 shows that the combined sequence $\left\{z_{n}\right\} \cup\left\{w_{n}\right\}$ has logarithmic exponent of convergence equal to one and the sequence is uniformly logarithmically $q$-separated.

To get an analogue of Lemma 3, we first modify [13, Thm. 3.1] to the case of finite logarithmic order. In the following statement, special attention has been paid to those points $z_{n}$ that are near the origin, as well as to minor monotonicity issues in [13, (3.2)].

Lemma 8 Suppose the following assumptions hold:

(a) $\left\{z_{n}\right\}$ is a sequence of distinct non-zero points in $\mathbb{C}$ with $\lambda_{\log }<\infty$.

(b) $\left\{c_{n}\right\}$ is a sequence of non-zero points in $\mathbb{C}$, not necessarily distinct.

(c) There exists a continuous function $g:[e, \infty) \rightarrow[1, \infty)$ such that $\log \left|c_{n}\right| / \log \left|z_{n}\right|$ $\leq g\left(\left|z_{n}\right|\right)$ for all $\left|z_{n}\right| \geq e$.

(d) Given $\alpha>1,\left\{q_{n}\right\}$ is a sequence such that each $q_{n} \geq 0$ is the smallest integer satisfying

$$
q_{n} \geq \max \left\{\alpha \frac{\log \left|c_{n}\right|}{\log \left|z_{n}\right|}, \frac{\log \frac{\left|c_{n}\right|}{n}}{\log \left|z_{n}\right|}+1\right\}
$$

for $\left|z_{n}\right|>e$, and set $q_{n}=0$ otherwise.

Then

$$
H(z)=\sum_{n=1}^{\infty} \frac{c_{n}}{z-z_{n}}\left(\frac{z}{z_{n}}\right)^{q_{n}}
$$

is meromorphic in $\mathbb{C}$, and has simple poles exactly at the points $z_{n}$ with residue $c_{n}$. Moreover, we have the growth estimates

$$
\lambda_{\log }+1 \leq \rho_{\log }(H) \leq \max \left\{\lambda_{\log }+1, \limsup _{r \rightarrow \infty} \frac{\log G(\alpha r)}{\log \log r}+1\right\},
$$

where $G(x)=\max _{e \leq t \leq x} g(t)$ is a non-decreasing function for $x \geq e$. 
Proof Without loss of generality, we may suppose that $\left\{z_{n}\right\}$ is ordered according to increasing moduli. Moreover, $G(x)$ is a well defined non-decreasing function by continuity.

Let $\beta=\sqrt[3]{\alpha}(>1)$ and $e<R<\infty$. Suppose that $|z|=r \leq R$, and write

$$
\begin{aligned}
H(z) & =\sum_{\left|z_{n}\right| \leq \beta R} \frac{c_{n}}{z-z_{n}}\left(\frac{z}{z_{n}}\right)^{q_{n}}+\sum_{\left|z_{n}\right|>\beta R} \frac{c_{n}}{z-z_{n}}\left(\frac{z}{z_{n}}\right)^{q_{n}} \\
& =: S_{1}(z)+S_{2}(z) .
\end{aligned}
$$

The expression $S_{1}(z)$ in (7.5) is a finite sum, and therefore it represents a rational meromorphic function in $\mathbb{C}$. Hence, in order to prove that $H(z)$ is meromorphic in $\mathbb{C}$, it suffices to show that $S_{2}(z)$ converges uniformly. But this can be done analogously as in [13, p. 293] by making use of the fact that the genus of $\left\{z_{n}\right\}$ is $p=0$.

Obviously, all poles $z_{n}$ of $H(z)$ are simple and have residue $c_{n}$.

The inequality $\lambda_{\log }+1 \leq \rho_{\log }(H)$ being clear from $N(r, H) \leq T(r, H)$, it remains to prove the second inequality in (7.4). This culminates in estimating $S_{1}(z)$. As $q_{n} \geq 0$ is the smallest integer satisfying (7.2), it follows that

$$
\begin{aligned}
q_{n} & \leq \alpha\left(\frac{\log \left|c_{n}\right|}{\log \left|z_{n}\right|}+1\right)+1 \leq \alpha\left(g\left(\left|z_{n}\right|\right)+1\right)+1 \\
& \leq \alpha\left(G\left(\left|z_{n}\right|\right)+1\right)+1, \quad\left|z_{n}\right| \geq e .
\end{aligned}
$$

Proceeding as in [13, p. 294] (but replacing $g$ with $G$ to ensure monotonicity), we can find a constant $C>0$ such that

$$
\left|S_{1}(z)\right| \leq C n\left(\beta^{2} R\right)(\log R)^{\beta} R^{\alpha(G(\beta R)+1)},
$$

provided that $|z| \leq R$ and $|z| \notin E \cup[0,1]$, where $E \subset[1, \infty)$ has finite logarithmic measure. Let $P$ be the canonical product associated with the sequence $\left\{z_{n}\right\}$, and hence $\rho_{\log }(P)=\lambda_{\log }+1$. Then $P H$ is entire, and

$$
|P(z) H(z)| \leq C M(r, P)\left(n\left(\beta^{2} R\right)(\log R)^{\beta} R^{\alpha(G(\beta R)+1)}+1\right),
$$

provided that $|z| \leq R$ and $|z| \notin E \cup[0,1]$. Taking now $R=\beta^{2} r$ and applying [8, Lem. 5], we see that there exists an $r_{0}=r_{0}(\beta)>0$ such that

$$
M(r, P H) \leq C M(\beta r, P)\left(n\left(\beta^{4} r\right)\left(\log \left(\beta^{2} r\right)\right)^{\beta}\left(\beta^{2} r\right)^{\alpha(G(\alpha r)+1)}+1\right)
$$

for all $r \geq r_{0}$. Since $\log n(r)=O(\log \log r)$, we deduce that

$$
\rho_{\log }(P H) \leq \max \left\{\lambda \log +1, \limsup _{r \rightarrow \infty} \frac{\log G(\alpha r)}{\log \log r}+1\right\} .
$$


The second inequality in (7.4) follows from $\rho_{\log }(H) \leq \max \left\{\rho_{\log }(P), \rho_{\log }(P H)\right\}$, which holds by using standard reasoning.

Lemma 9 Suppose that $\left\{z_{n}\right\}$ is an infinite sequence of non-zero points in $\mathbb{C}$ with $\lambda \log _{\log }<$ $\infty$, and that $\left\{z_{n}\right\}$ is uniformly logarithmically $q$-separated for some $q \geq 0$ (and $C>0)$. Let $\left\{\sigma_{n}\right\}$ be an infinite sequence of points in $\mathbb{C}$, not necessarily distinct, and let $h:[0, \infty) \rightarrow[1, \infty)$ be a continuous and non-decreasing function such that $\left|\sigma_{n}\right| \leq h\left(\left|z_{n}\right|\right)$ for $n \in \mathbb{N}$.

Then there exists an entire function $A(z) \not \equiv 0$ such that

$$
A\left(z_{n}\right)=\sigma_{n}, \quad n \in \mathbb{N},
$$

and, for any given $\alpha>1$,

$$
\rho_{\log }(A) \leq \max \left\{\lambda_{\log }+1, \limsup _{r \rightarrow \infty} \frac{\log F(\alpha r)}{\log \log r}+1\right\},
$$

where $F(x)=\max _{e \leq t \leq x} \frac{\log \left(h(t) e^{C(\log (1+t))^{q}}\right)}{\log t}$ is a non-decreasing function for $x \geq e$.

Proof Without loss of generality, we may suppose that $\left\{z_{n}\right\}$ is ordered according to increasing moduli. Moreover, $F(x)$ is well defined and non-decreasing by continuity. Let $P(z)$ be the canonical product having $\left\{z_{n}\right\}$ as its zero sequence. By uniform logarithmic $q$-separation, the points $z_{n}$ are simple, and hence $1 / P^{\prime}\left(z_{n}\right) \in \mathbb{C}$ for any $n$.

(a) Suppose that $\sigma_{n} \neq 0$ for all $n$. If $H(z)$ denotes the Mittag-Leffler series in (7.3), where $c_{n}=\sigma_{n} / P^{\prime}\left(z_{n}\right)$, then $A=P H$ is entire and satisfies (7.6). Hence it suffices to prove (7.7). By the assumptions, there exists a constant $C_{1}>0$ such that

$$
\left|c_{n}\right| \leq C_{1}\left|z_{n}\right| h\left(\left|z_{n}\right|\right) e^{C\left(\log \left(1+\left|z_{n}\right|\right)\right)^{q}}, \quad n \in \mathbb{N} .
$$

Thus

$$
\frac{\log \left|c_{n}\right|}{\log \left|z_{n}\right|} \leq \frac{\log \left(h\left(\left|z_{n}\right|\right) e^{C\left(\log \left(1+\left|z_{n}\right|\right)\right)^{q}}\right)}{\log \left|z_{n}\right|}+C_{2}, \quad\left|z_{n}\right| \geq e,
$$

where $C_{2}>0$ is a constant. Choosing

$$
g(t)=\frac{\log \left(h(t) e^{C(\log (1+t))^{q}}\right)}{\log t}+C_{2}
$$

for $t \geq e$, we deduce by Lemma 8 that

$$
\rho_{\log }(H) \leq \max \left\{\lambda \log +1, \limsup _{r \rightarrow \infty} \frac{\log F(\alpha r)}{\log \log r}+1\right\}
$$


where $\alpha>1$ and

$$
F(x)=\max _{e \leq t \leq x} \frac{\log \left(h(t) e^{C(\log (1+t))^{q}}\right)}{\log t} .
$$

The assertion (7.7) then follows from $\rho_{\log }(A) \leq \max \left\{\rho_{\log }(P), \rho_{\log }(H)\right\}$.

(b) Suppose that $\sigma_{n}=0$ for at least one $n$. If $\sigma_{n}=0$ for all $n$, we may choose $A(z)=P(z)$, in which case (7.6) and (7.7) clearly hold. Hence we may suppose that $\sigma_{n}=0$ for at least one index $n$ but not for all $n$. But this case is analogous to Part (b) in the proof of Lemma 3.

\section{Proof of Theorem 3}

Anderson and Clunie proved the following result [1, Thm. 2]: Given any continuous function $\varphi(r)$ tending monotonically to infinity as $r \rightarrow \infty$, no matter how slowly, there exists an entire function $f$ such that $\log M(r, f)=O\left(\varphi(r)(\log r)^{2}\right)$ and $\delta_{V}(0, f)=$ 1.

The solution $f$ to the problem above has real zeros with multiplicities tending to infinity. Moreover, the proof given in [1] is based upon a technical restriction $\varphi(r)=O(\log r)$, which yields $\rho_{\log }(f) \in(2,3)$. Next we present a modification of the Anderson-Clunie reasoning such that the solution $f$ has only simple zeros, and hence it is suitable for being a solution of (1.2). In addition, $f$ has arbitrary pre-determined logarithmic order on $(2, \infty)$.

Lemma 10 For every $\rho \in(2, \infty)$ there exists an entire function $f$ with simple zeros such that $\rho_{\log }(f)=\rho$ and $\delta_{V}(0, f)=1$.

Proof Given $m \in \mathbb{N}$ and $b>0$, define a polynomial

$$
P_{m, b}(z)=\prod_{k=1}^{m}\left(1-\frac{z}{b e^{i \varphi_{m, k}}}\right)
$$

where the arguments $\varphi_{m, k}$ are chosen such that the zeros $b e^{i \varphi_{m, k}}$ of $P_{m, b}$ lie symmetrically in $3 \pi / 4 \leq \arg (z) \leq 5 \pi / 4$ and on the circle $|z|=b$ as follows:

$$
\begin{aligned}
& m=1: \varphi_{1,1}=\pi \\
& m=2: \varphi_{2,1}=\frac{3 \pi}{4}, \varphi_{2,2}=\frac{5 \pi}{4}, \\
& m=3: \varphi_{3,1}=\frac{3 \pi}{4}, \varphi_{3,2}=\pi, \varphi_{3,3}=\frac{5 \pi}{4}, \\
& m=4: \varphi_{4,1}=\frac{3 \pi}{4}, \varphi_{4,2}=\frac{11 \pi}{12}, \varphi_{4,3}=\frac{13 \pi}{12}, \varphi_{4,4}=\frac{5 \pi}{4},
\end{aligned}
$$


and, in general, for any integer $l \geq 1$,

$$
\begin{aligned}
& m=2 l+1: \varphi_{2 l+1, i}=\pi-\frac{\pi}{4} \cdot \frac{l-i+1}{l}, \quad i=1, \ldots, 2 l+1, \\
& m=2 l: \varphi_{2 l, i}=\pi-\frac{\pi}{4} \cdot \frac{2(l-i)+1}{2 l-1}, \quad i=1, \ldots, 2 l .
\end{aligned}
$$

If $m$ is even, then all zeros on $|z|=b$ are pairwise complex conjugate numbers. If $m$ is odd, then precisely one of the zeros on $|z|=b$ lies on the negative real axis, while the rest are pairwise complex conjugates. Thus, if $z \in \mathbb{R}$, we have $P_{m, b}(z) \in \mathbb{R}$ due to the elementary identity $\zeta \bar{\zeta}=|\zeta|^{2}$. In particular, $P_{m, b}$ is a real polynomial, and $\log P_{m, b}(z)$ is analytic in $-3 \pi / 4<\arg (z)<3 \pi / 4$.

If $P_{m, b}$ has a real zero at $\zeta \in \mathbb{R}_{-}$, then the modulus

$$
\left|1-\frac{z}{\zeta}\right|=\frac{|\zeta-z|}{|\zeta|}
$$

on any circle $|z|=r$ is maximal when $z=r$. Suppose then that $\zeta, \bar{\zeta}$ is any pair of conjugate zeros of $P_{m, b}$. Then the modulus

$$
\left|\left(1-\frac{z}{\zeta}\right)\left(1-\frac{z}{\bar{\zeta}}\right)\right|=\frac{|\zeta-z||\bar{\zeta}-z|}{|\zeta|^{2}}
$$

on any circle $|z|=r$ is maximal when $z$ is simultaneously as far as possible from both points $\zeta, \bar{\zeta}$ that lie in $3 \pi / 4 \leq \arg (w) \leq 5 \pi / 4$, that is, when $z=r$. We repeat this reasoning for all zeros of $P_{m, b}$, and conclude that

$$
M\left(r, P_{m, b}\right)=P_{m, b}(r)
$$

Moreover, a simple geometric reasoning yields the growth estimates

$$
\left(1+\frac{r^{2}}{b^{2}}\right)^{m / 2}=\left(\frac{\sqrt{b^{2}+r^{2}}}{b}\right)^{m} \leq P_{m, b}(r) \leq\left(1+\frac{r}{b}\right)^{m}
$$

Next we define two non-decreasing sequences $\left\{b_{n}\right\}$ and $\left\{c_{n}\right\}$ of positive integers by setting $b_{1}=1=c_{1}$, and

$b_{n}=\exp \left(\left(\sum_{j=1}^{n-1} c_{j}\right)^{2 /(\rho-2)}\right), \quad c_{n}=\left\lfloor\left(\log b_{n}\right)^{\rho-1}\right\rfloor=\left\lfloor\left(\sum_{j=1}^{n-1} c_{j}\right)^{2(\rho-1) /(\rho-2)}\right\rfloor$,

for $n \geq 2$, where $\lfloor x\rfloor$ denotes the integer part of $x$. This definition corresponds to the choice of points $b_{n}, c_{n}$ in [1] where $\varphi(r)=(\log r)^{\rho-2}$. We define a formal canonical 
product $f$ in terms of the polynomial factors in (8.1) as

$$
f(z)=\prod_{n=1}^{\infty} P_{c_{n}, b_{n}}(z),
$$

and prove that this function has the required properties.

For $r>1$ there exists an integer $n$ such that $b_{n}<r \leq b_{n+1}$. If $n(r)$ denotes the number of zeros of $f$ in $|z|<r$, counting multiplicities, then

$$
\begin{aligned}
n(r) & =n\left(b_{n}\right)=\sum_{j=1}^{n} c_{j}=c_{n}+\left(\log b_{n}\right)^{(\rho-2) / 2} \\
& \leq 2\left(\log b_{n}\right)^{\rho-1} \leq 2(\log r)^{\rho-1} .
\end{aligned}
$$

Thus the zeros of the formal product $f$ have logarithmic exponent of convergence at most $\leq \rho-1$, so that $f$ is entire, and, in fact, $\rho_{\log }(f) \leq \rho$, see [4]. Let $\beta=$ $2(\rho-1) /(\rho-2)>2$ for short. The auxiliary function

$$
g(x)=\frac{(1+x)^{\beta}}{1+x^{\beta}}, \quad x \geq 0,
$$

is increasing on $[0,1]$ and decreasing on $[1, \infty)$. Since $g(0)=1$ and since $g(x) \rightarrow 1$ as $x \rightarrow \infty$, we have $g(x) \geq 1$ for all $x \geq 0$, that is,

$$
(1+x)^{\beta} \geq 1+x^{\beta}, \quad x \geq 0 .
$$

Therefore, we have $c_{1}=c_{2}=1, c_{3}=\left\lfloor 2^{\beta}\right\rfloor \geq\left\lfloor 2^{2}\right\rfloor=4$, and

$$
\begin{aligned}
c_{n} & \geq\left\lfloor\left(1+c_{n-1}\right)^{\beta}\right\rfloor \geq\left\lfloor 1+c_{n-1}^{\beta}\right\rfloor \geq c_{n-1}^{\beta} \geq\left\lfloor\left(1+c_{n-2}\right)^{\beta^{2}}\right\rfloor \\
& \geq\left\lfloor 1+c_{n-2}^{\beta^{2}}\right\rfloor \geq c_{n-2}^{\beta^{2}} \geq \cdots \geq c_{3}^{\beta^{n-3}} \geq 4^{\beta^{n-3}}, \quad n \geq 4 .
\end{aligned}
$$

Let $\mu>\rho-1$, so that $\sigma:=\mu /(\rho-1)-1>0$. Since

$$
\begin{aligned}
& \sum_{n=4}^{\infty} \frac{c_{n}}{\left(\log b_{n}\right)^{\mu}} \leq \sum_{n=4}^{\infty} \frac{c_{n}}{\left(\left\lfloor\left(\log b_{n}\right)^{\rho-1}\right\rfloor\right)^{\mu /(\rho-1)}}=\sum_{n=4}^{\infty} \frac{1}{c_{n}^{\sigma}} \leq \sum_{n=4}^{\infty} 4^{-\sigma \beta^{n-3}}<\infty, \\
& \sum_{n=2}^{\infty} \frac{c_{n}}{\left(\log b_{n}\right)^{\rho-1}} \geq \sum_{n=2}^{\infty} \frac{c_{n}}{\left\lfloor\left(\log b_{n}\right)^{\rho-1}\right\rfloor+1}=\sum_{n=2}^{\infty} \frac{c_{n}}{c_{n}+1}=\infty,
\end{aligned}
$$

it follows that the logarithmic exponent of convergence of the zero sequence of $f$ is equal to $\rho-1$, and thus $\rho_{\log }(f)=\rho$, see [4].

It remains to prove that $\delta_{V}(0, f)=1$, which is equivalent to

$$
\liminf _{r \rightarrow \infty} \frac{N(r, 1 / f)}{T(r, f)}=0 .
$$


It suffices to show that

$$
\lim _{n \rightarrow \infty} \frac{N\left(b_{n}, 1 / f\right)}{T\left(b_{n}, f\right)}=0
$$

For $n \geq 2$, we have

$$
\begin{aligned}
N\left(b_{n}, 1 / f\right) & =\int_{0}^{b_{n}} \frac{n(t)}{t} d t \leq n\left(b_{n-1}\right) \int_{1}^{b_{n}} \frac{d t}{t} \\
& =\left(\sum_{j=1}^{n-1} c_{j}\right) \log b_{n}=\left(\log b_{n}\right)^{\rho / 2} .
\end{aligned}
$$

Estimating the characteristic function from below requires more work. To begin with, we observe that the representation $[1,(5.3)]$ for $\log M(r, f)$ is not valid in our case. However, we see by means of (8.2) and (8.3) that

$$
\log M(r, f)=\log f(r)=\sum_{n=1}^{\infty} \log P_{c_{n}, b_{n}}(r) \geq \sum_{n=1}^{\infty} \frac{c_{n}}{2} \log \left(1+\frac{r^{2}}{b_{n}^{2}}\right)
$$

For a fixed $r>0$ and all $t>r$, we deduce by (8.5) and L'Hospital's rule that

$$
0 \leq n(t) \log \left(1+\frac{r^{2}}{t^{2}}\right) \leq 2 \frac{\log \left(1+\frac{r^{2}}{t^{2}}\right)}{(\log t)^{-(\rho-1)}} \sim \frac{4 r^{2}}{\rho-1} \cdot \frac{(\log t)^{\rho}}{r^{2}+t^{2}} \rightarrow 0
$$

as $t \rightarrow \infty$. Therefore, Riemann-Stieltjes integration and integration by parts give us

$$
\log M(r, f) \geq \frac{1}{2} \int_{0}^{\infty} \log \left(1+\frac{r^{2}}{t^{2}}\right) d n(t)=r^{2} \int_{0}^{\infty} \frac{n(t) d t}{t\left(t^{2}+r^{2}\right)}
$$

Using the well-known inequality [11, p. 18]

$$
\log M(r, f) \leq 3 T(2 r, f), \quad r>0,
$$

we then deduce that

$$
T(r, f) \geq \frac{r^{2}}{12} \int_{0}^{\infty} \frac{n(t) d t}{t\left(t^{2}+\frac{r^{2}}{4}\right)} \geq \frac{n\left(b_{n}\right) r^{2}}{12} \int_{b_{n}}^{2 b_{n}} \frac{d t}{t\left(t^{2}+\frac{r^{2}}{4}\right)}
$$

where

$$
n\left(b_{n}\right)=\sum_{j=1}^{n} c_{j} \geq c_{n}=\left\lfloor\left(\log b_{n}\right)^{\rho-1}\right\rfloor \geq\left(\log b_{n}\right)^{\rho-1}-1
$$


Substituting $r=b_{n}$ and $t=b_{n} u$ in (8.9), it follows that

$$
T\left(b_{n}, f\right) \geq \frac{1}{12}\left(\left(\log b_{n}\right)^{\rho-1}-1\right) \int_{1}^{2} \frac{d u}{u\left(u^{2}+\frac{1}{4}\right)} \geq \frac{1}{40}\left(\left(\log b_{n}\right)^{\rho-1}-1\right) .
$$

Combining this with (8.8) finally gives (8.7), because $\rho>2$.

It is not necessary for $k, n$ in Lemma 5 to be integers. We will state, without proof, the following analogue of Lemma 5 needed later on.

Lemma 11 For $0<A<B$ and $\gamma>0$, we have the inequalities

$$
\begin{array}{ll}
\gamma(B-A) B^{\gamma-1} \leq B^{\gamma}-A^{\gamma} \leq \gamma(B-A) A^{\gamma-1}, & 0<\gamma \leq 1, \\
\gamma(B-A) A^{\gamma-1} \leq B^{\gamma}-A^{\gamma} \leq \gamma(B-A) B^{\gamma-1}, & 1 \leq \gamma<\infty .
\end{array}
$$

It turns out that the zero sequence of the function $f$ in Lemma 10 is separated in the following sense.

Lemma 12 The zero sequence of the function $f$ in (8.4) is uniformly logarithmically $q$-separated for every $q>\rho-1$.

Proof Let $\left\{z_{n}\right\}$ denote the zeros of $f$ organized first according to increasing modulus and then, on each circle $|z|=b_{j}$, according to increasing argument on $[3 \pi / 4,5 \pi / 4]$. This fixes $\left\{z_{n}\right\}$ uniquely. Since $\sum_{n}\left|z_{n}\right|^{-1}<\infty$ and since $\left|z_{n}\right| \geq e$ for $n \geq 2$, we observe that

$$
\prod_{n \geq 2}\left|1-\frac{z_{1}}{z_{n}}\right| \geq \prod_{n \geq 2}\left(1-\frac{1}{\left|z_{n}\right|}\right) \geq C_{0}>0 .
$$

If $k \geq 2$, we have $\left|z_{k}\right|=b_{i}$ for some $2 \leq i \leq k$, and

$$
\begin{aligned}
\prod_{n \neq k}\left|1-\frac{z_{k}}{z_{n}}\right| & =\prod_{\left|z_{n}\right|<b_{i}}\left|1-\frac{z_{k}}{z_{n}}\right| \prod_{\left|z_{n}\right|>b_{i}}\left|1-\frac{z_{k}}{z_{n}}\right| \prod_{\left|z_{n}\right|=b_{i}, z_{n} \neq z_{k}}\left|1-\frac{z_{k}}{z_{n}}\right| \\
& =: P_{1}(k) P_{2}(k) P_{3}(k),
\end{aligned}
$$

where we set $P_{3}(2)=1$ because $z_{n}$ is on the circle $|z|=b_{2}$ only when $n=2$. The products $P_{1}(k), P_{2}(k), P_{3}(k)$ converge for any finite $k$, so it suffices to estimate them for arbitrary large values of $k$ (or for arbitrarily large values of $i$ due to $\left|z_{k}\right|=b_{i}$ ).

Estimate for $P_{1}(k)$ : We have

$$
P_{1}(k) \geq \prod_{\left|z_{n}\right|<b_{i}}\left(\left|\frac{z_{k}}{z_{n}}\right|-1\right)=\prod_{m=1}^{i-1}\left(\frac{b_{i}}{b_{m}}-1\right)^{c_{m}} \geq \prod_{m=1}^{i-1}\left(\frac{b_{i}}{b_{i-1}}-1\right)^{c_{m}} .
$$


If $i=2$, we get $P_{1}(k) \geq e-1>1$, while if $i \geq 3$, we use the inequality $e^{x}-1 \geq x$, and obtain

$$
P_{1}(k) \geq \prod_{m=1}^{i-1}\left(\left(\sum_{j=1}^{i-1} c_{j}\right)^{\gamma}-\left(\sum_{j=1}^{i-2} c_{j}\right)^{\gamma}\right)^{c_{m}}
$$

where $\gamma=2 /(\rho-2)$. If $\gamma=1$, we clearly have $P_{1}(k) \geq 1$. If $\gamma>1$, then (8.11) gives

$$
P_{1}(k) \geq \prod_{m=1}^{i-1}\left(\gamma\left(\sum_{j=1}^{i-2} c_{j}\right)^{\gamma-1} c_{i-1}\right)^{c_{m}} \geq \prod_{m=1}^{i-1}\left(\gamma c_{1}^{\gamma}\right)^{c_{m}} \geq 1 .
$$

If $0<\gamma<1$, then from (8.10) we have

$$
\begin{aligned}
e^{\left(\log \left(1+\left|z_{k}\right|\right)\right)^{q}} P_{1}(k) & \geq e^{\left(\log b_{i}\right)^{q}} \prod_{m=1}^{i-1}\left(\gamma\left(\sum_{j=1}^{i-1} c_{j}\right)^{\gamma-1} c_{i-1}\right)^{c_{m}} \\
& \geq \exp \left(\left(\sum_{j=1}^{i-1} c_{j}\right)^{\gamma q}-\sum_{m=1}^{i-1} c_{m} \log \left(\frac{1}{\gamma}\left(\sum_{j=1}^{i-1} c_{j}\right)^{1-\gamma}\right)\right)
\end{aligned}
$$

The function $x \mapsto x^{\gamma q}-x \log \left(x^{1-\gamma} / \gamma\right)$ is eventually increasing and unbounded for every $q>1 / \gamma$. Summarizing, for any $\gamma>0$ there exists a constant $C_{1}=C_{1}(\gamma, q)>$ 0 such that

$$
e^{\left(\log \left(1+\left|z_{k}\right|\right)\right)^{q}} P_{1}(k) \geq C_{1}, \quad q>\frac{\rho-2}{2}, k \geq 2 .
$$

Estimate for $P_{2}(k)$ : Clearly $j \leq c_{j} \leq c_{j+1}$ for every $j \geq 3$, and so

$$
\begin{aligned}
c_{n} & \leq\left(\sum_{j=1}^{n-1} c_{j}\right)^{\beta} \leq\left((n-1) c_{n-1}\right)^{\beta} \leq c_{n-1}^{2 \beta} \\
& \leq\left(\sum_{j=1}^{n-2} c_{j}\right)^{2 \beta^{2}} \leq\left((n-2) c_{n-2}\right)^{2 \beta^{2}} \leq c_{n-2}^{(2 \beta)^{2}} \\
& \leq \cdots \leq c_{3}^{(2 \beta)^{n-3}} \leq\left(2^{\beta}\right)^{(2 \beta)^{n-3}} \leq 2^{(2 \beta)^{n-2}}, \quad n \geq 5 .
\end{aligned}
$$


Suppose that $\gamma=1$. For $m \geq i+1 \geq 5$, the estimate (8.6) yields

$$
\frac{b_{i}}{b_{m}}=\exp \left(-\sum_{j=i}^{m-1} c_{j}\right) \leq \exp \left(-c_{m-1}\right) \leq \exp \left(-4^{\beta^{m-4}}\right)
$$

In particular, $b_{i} / b_{m} \leq 1 / e<1 / 2$ for all $m \geq i+1 \geq 5$. Thus, using $\log (1-x) \geq-2 x$ for $x \in[0,1 / 2]$, we have

$$
\begin{aligned}
P_{2}(k) & \geq \prod_{\left|z_{n}\right|>b_{i}}\left(1-\left|\frac{z_{k}}{z_{n}}\right|\right)=\prod_{m=i+1}^{\infty}\left(1-\frac{b_{i}}{b_{m}}\right)^{c_{m}} \\
& =\exp \left(\sum_{m=i+1}^{\infty} c_{m} \log \left(1-\frac{b_{i}}{b_{m}}\right)\right) \geq \exp \left(-2 \sum_{m=i+1}^{\infty} c_{m} \cdot \frac{b_{i}}{b_{m}}\right) .
\end{aligned}
$$

By combining (8.14) and (8.15), we deduce that

$$
P_{2}(k) \geq \exp \left(-2 \sum_{m=5}^{\infty} \exp \left((2 \beta)^{m-2} \log 2-4^{\beta^{m-4}}\right)\right)
$$

Since $(2 \beta)^{m-2} \log 2-4^{\beta^{m-4}} \leq-4^{\beta^{m-5}}$ for all $m$ large enough, the series above converges. Suppose then that $\gamma>1$. We multiply both sides of the first inequality in (8.11) by the constant -1 , and use the resulting inequality together with $m \geq i+1$ to deduce that

$$
\begin{aligned}
\frac{b_{i}}{b_{m}} & =\exp \left(\left(\sum_{j=1}^{i-1} c_{j}\right)^{\gamma}-\left(\sum_{j=1}^{m-1} c_{j}\right)^{\gamma}\right) \\
& \leq \exp \left(-\gamma\left(\sum_{j=1}^{i-1} c_{j}\right)^{\gamma-1} \cdot \sum_{j=i}^{m-1} c_{j}\right) \\
& \leq \exp \left(-\gamma c_{1}^{\gamma-1} c_{m-1}\right) \leq \exp \left(-c_{m-1}\right) .
\end{aligned}
$$

Next we use (8.6) to obtain

$$
\frac{b_{i}}{b_{m}} \leq \exp \left(-4^{\beta^{m-4}}\right)
$$

and then proceed in the same way as in the case $\gamma=1$. Finally, suppose that $0<\gamma<1$. We multiply both sides of the first inequality in (8.10) by the constant -1 , and use the 
resulting inequality together with $m \geq i+1$ to deduce that

$$
\begin{aligned}
\frac{b_{i}}{b_{m}} & \leq \exp \left(-\gamma\left(\sum_{j=1}^{m-1} c_{j}\right)^{\gamma-1} \cdot \sum_{j=i}^{m-1} c_{j}\right) \leq \exp \left(-\gamma c_{m-1}\left(\sum_{j=1}^{m-1} c_{j}\right)^{\gamma-1}\right) \\
& \leq \exp \left(-\gamma c_{m-1}\left((m-1) c_{m-1}\right)^{\gamma-1}\right)=\exp \left(-\gamma(m-1)^{\gamma-1} c_{m-1}^{\gamma}\right),
\end{aligned}
$$

where $m \geq i+1 \geq 5$. For $i$ large enough, $b_{i} / b_{m} \leq 1 / e<1 / 2$ clearly holds. Similarly as above,

$$
\begin{aligned}
P_{2}(k) & \geq \exp \left(-2 \sum_{m=i+1}^{\infty} c_{m} \cdot \frac{b_{i}}{b_{m}}\right) \\
& \geq \exp \left(-2 \sum_{m=5}^{\infty} \exp \left((2 \beta)^{m-2} \log 2-\gamma(m-1)^{\gamma-1} 4^{\gamma \beta^{m-4}}\right)\right) .
\end{aligned}
$$

Since $(2 \beta)^{m-2} \log 2-\gamma(m-1)^{\gamma-1} 4^{\gamma \beta^{m-4}} \leq-4^{\gamma \beta^{m-5}}$ for all $m$ large enough, the series above converges. Summarizing, for any $\gamma>0$ there exists a constant $C_{2}>0$ such that

$$
P_{2}(k) \geq C_{2}, \quad k \geq 2 .
$$

Estimate for $P_{3}(k)$ : Recall that $P_{3}(k)$ is undefined for $i=1$, and that we have set $P_{3}(k)=1$ for $i=2$, so suppose that $i \geq 3$. Now $c_{i} \geq c_{3} \geq 4$. There are $c_{i}-1$ factors in $P_{3}(k)$. The distance between two consecutive zeros $z_{n}$ on the circle $|z|=b_{i}$ is $2 b_{i} \sin \left(\pi /\left(4\left(c_{i}-1\right)\right)\right)$. Using $\sin x \geq x / \sqrt{2}$ for $0<x<\pi / 4$, we get

$$
\begin{aligned}
P_{3}(k) & =\prod_{\left|z_{n}\right|=b_{i}, z_{n} \neq z_{k}} \frac{\left|z_{n}-z_{k}\right|}{b_{i}} \geq\left(2 \sin \frac{\pi}{4\left(c_{i}-1\right)}\right)^{c_{i}-1} \\
& \geq\left(\frac{\sqrt{2} \pi}{4\left(c_{i}-1\right)}\right)^{c_{i}-1} \geq\left(\frac{1}{c_{i}-1}\right)^{c_{i}-1} \geq\left(\frac{1}{c_{i}}\right)^{c_{i}},
\end{aligned}
$$

and so

$$
\begin{aligned}
e^{\left(\log \left(1+\left|z_{k}\right|\right)\right)^{q}} P_{3}(k) & \geq \exp \left(\left(\log b_{i}\right)^{q}-c_{i} \log c_{i}\right) \\
& \geq \exp \left(\left(\log b_{i}\right)^{q}-(\rho-1)\left(\log b_{i}\right)^{\rho-1} \log \log b_{i}\right) .
\end{aligned}
$$

The function $x \mapsto(\log x)^{q}-(\rho-1)(\log x)^{\rho-1} \log \log x$ is eventually positive for every $q>\rho-1$. Thus there exists a constant $C_{3}=C_{3}(q)>0$ such that

$$
e^{\left(\log \left(1+\left|z_{k}\right|\right)\right)^{q}} P_{3}(k) \geq C_{3}, \quad q>\rho-1, k \geq 2 .
$$


Final conclusion We complete the proof of Lemma 12 by combining (8.12) with (8.13), (8.16) and (8.17).

Proof of Theorem 3 After these preparations, the actual proof of Theorem 3 is now easy. Let $f$ be the function in (8.4). By Lemma 10, $f$ has the required properties for the solution of (1.2). Further, by Lemma 12, the zero sequence of $f$ is uniformly logarithmically $q$-separated for any $q>\rho-1$. Since $\rho=\rho_{\log }(f)=\rho_{\log }\left(f^{\prime \prime}\right)$, the target sequence $\left\{\sigma_{n}\right\}$ in (3.1) can be estimated as follows: For every $\sigma>\rho$ there exist constants $C>0$ and $C_{1}>0$ such that

$$
\left|\sigma_{n}\right| \leq C_{1}\left|z_{n}\right| \exp \left(\left(\log \left(1+\left|z_{n}\right|\right)\right)^{\sigma}+C\left(\log \left(1+\left|z_{n}\right|\right)\right)^{q}\right), \quad n \in \mathbb{N}
$$

Using Lemma 9, we conclude that there exists an entire function $A(z)$ satisfying (3.1) such that $\rho_{\log }(A) \leq \max \left\{\rho_{\log }(f), q\right\}$, where we may suppose that $q \leq \rho_{\log }(f)$.

Finally, we define the entire coefficient $B(z)$ by (3.3). It is easy to see that $A(z)$ must be transcendental. For if $A(z)$ is a polynomial, then

$$
T(r, B)=m(r, B)=m\left(r, \frac{f^{\prime \prime}}{f}+A \frac{f^{\prime}}{f}\right)=O(\log r),
$$

so that $B(z)$ is also a polynomial. But this leads to a contradiction as any transcendental solution must be of positive usual order in the case of polynomial coefficients [9]. Thus

$$
\liminf _{r \rightarrow \infty} \frac{T(r, A)}{\log r}=\infty \text {. }
$$

Using the lemma on the logarithmic derivative to get

$$
|B(z)| \leq\left|\frac{f^{\prime \prime}(z)}{f(z)}\right|+|A(z)| \frac{f^{\prime}(z)}{f(z)} \mid,
$$

we then obtain $\rho_{\log }(B) \leq \rho_{\log }(A)$. Thus it remains to show that $B(z) \not \equiv 0$. Suppose on the contrary that $B(z) \equiv 0$, in which case (1.2) reduces to $f^{\prime \prime}+A(z) f^{\prime}=0$. Then either $f$ is a polynomial or $\rho(f) \geq 1$, which are both impossible. This completes the proof.

Acknowledgements Open access funding provided by University of Eastern Finland (UEF) including Kuopio University Hospital. All authors were supported by the Academy of Finland Project \#268009. The second author was supported by the Magnus Ehrnrooth Foundation and the Vilho, Yrjö and Kalle Väisälä Foundation of the Finnish Academy of Science and Letters. The third author was supported by the National Natural Science Foundation of China (No. 11771090 and No. 11971288), the Program of Visiting Scholars at Chern Institute of Mathematics and Shantou University SRFT (NTF18029).

Open Access This article is licensed under a Creative Commons Attribution 4.0 International License, which permits use, sharing, adaptation, distribution and reproduction in any medium or format, as long as you give appropriate credit to the original author(s) and the source, provide a link to the Creative Commons licence, and indicate if changes were made. The images or other third party material in this article are included in the article's Creative Commons licence, unless indicated otherwise in a credit line to the material. If material is not included in the article's Creative Commons licence and your intended use is not permitted 
by statutory regulation or exceeds the permitted use, you will need to obtain permission directly from the copyright holder. To view a copy of this licence, visit http://creativecommons.org/licenses/by/4.0/.

\section{References}

1. Anderson, J.M., Clunie, J.: Slowly growing meromorphic functions. Comment. Math. Helv. 40, 267280 (1966)

2. Bank, S.: A note on the zero-sequences of solutions of linear differential equations. Results Math. 13, $1-12$ (1988)

3. Berg, C., Pedersen, H.: Logarithmic order and type of indeterminate moment problems. With an appendix by Walter Hayman. Difference equations, special functions and orthogonal polynomials, pp. 51-79. World Sci. Publ., Hackensack (2007)

4. Chern, P.T.-Y.: On meromorphic functions with finite logarithmic order. Trans. Am. Math. Soc. 358(2), 473-489 (2006)

5. Edrei, A., Fuchs, W.: On the growth of meromorphic functions with several deficient values. Trans. Am. Math. Soc. 93, 292-328 (1959)

6. Goldberg, A.A., Ostrovskii, I.V.: Value distribution of meromorphic functions. Translated from the 1970 Russian original by Mikhail Ostrovskii. With an appendix by Alexandre Eremenko and James K. Langley. Translations of Mathematical Monographs, vol. 236. American Mathematical Society, Providence (2008)

7. Gröhn, J., Heittokangas, J.: New findings on the Bank-Sauer approach in oscillation theory. Constr. Approx. 35(3), 345-361 (2012)

8. Gundersen, G.G.: Finite order solutions of second order linear differential equations. Trans. Am. Math. Soc. 305(1), 415-429 (1988)

9. Gundersen, G.G., Steinbart, E.M., Wang, S.: The possible orders of solutions of linear differential equations with polynomial coefficients. Trans. Am. Math. Soc. 350(3), 1225-1247 (1998)

10. Havin, V.P., Nikolski, N.K. (eds.) Linear and Complex Analysis. Problem Book 3. Part II. Lecture Notes in Mathematics, vol. 1574. Springer, Berlin (1994)

11. Hayman, W.K.: Meromorphic Functions. Clarendon Press, Oxford (1964)

12. Hayman, W.K.: On the Valiron deficiencies of integral functions of infinite order. Ark. Mat. 10, 163-172 (1972)

13. Heittokangas, J., Laine, I.: Solutions of $f^{\prime \prime}+A(z) f=0$ with prescribed sequences of zeros. Acta Math. Univ. Comenianae LXXIV(2), 287-307 (2005)

14. Hyllengren, A.: Valiron deficient values for meromorphic functions in the plane. Acta Math. 124, 1-8 (1970)

15. Laine, I.: Nevanlinna Theory and Complex Differential Equations. Walter de Gruyter, Berlin (1993)

16. Levin, B.Ja.: Distribution of Zeros of Entire Functions. Translated from the Russian by R. P. Boas, J. M. Danskin, F. M. Goodspeed, J. Korevaar, A. L. Shields and H. P. Thielman. Revised edition. Translations of Mathematical Monographs, vol. 5. American Mathematical Society, Providence (1980)

17. Nevanlinna, R.: Le Théorème de Picard-Borel et la Théorie des Fonctions Méromorphes. GauthierVillars, Paris (1929)

18. Nevanlinna, R.: Analytic Functions. Translated from the second German edition by Phillip Emig. Die Grundlehren der mathematischen Wissenschaften, Band 162. Springer, New York (1970)

19. Steinmetz, N.: Zur Wertverteilung von Exponentialpolynomen. Manuscr. Math. 26(1-2), 155-167 (1978/1979)

20. Tsuji, M.: Potential Theory in Modern Function Theory. Reprinting of the 1959 original. Chelsea Publishing Co., New York (1975)

21. Valiron, G.: Sur les valeurs déficientes des fonctions algébroïdes méromorphes. J. Anal. Math. 1, 28-42 (1951). (French)

22. Wittich, H.: Neuere Untersuchungen über eindeutige analytische Funktionen, 2nd edn. Springer, Berlin (1968)

Publisher's Note Springer Nature remains neutral with regard to jurisdictional claims in published maps and institutional affiliations. 


\section{Affiliations}

\section{Gary G. Gundersen ${ }^{1}$ • Janne Heittokangas ${ }^{2,4} \cdot$ Zhi-Tao Wen $^{3,4}$}

Janne Heittokangas

janne.heittokangas@uef.fi

Gary G. Gundersen

ggunders@uno.edu

Zhi-Tao Wen

zhtwen@stu.edu.cn

1 Department of Mathematics, University of New Orleans, New Orleans, LA 70148, USA

2 Department of Physics and Mathematics, University of Eastern Finland, P.O. Box 111, 80101 Joensuu, Finland

3 Department of Mathematics, Shantou University, Daxue Road No. 243, Shantou 515063, China

4 Department of Mathematics, Taiyuan University of Technology, Yingze West Street, No. 79, Taiyuan 030024, China 